\title{
Investing in a Real World With Mean-Reverting Inflation
}

\author{
Arjan Berkelaar and Roy Kouwenberg* \\ Econometric Institute Report EI-9960/A \\ Econometric Institute \\ Faculty of Economics \\ Erasmus University Rotterdam \\ P.O.Box 1738, 3000 DR Rotterdam \\ The Netherlands \\ Department of Finance \\ Faculty of Economics \\ Erasmus University Rotterdam \\ P.O.Box 1738, 3000 DR Rotterdam \\ The Netherlands
}

\begin{abstract}
People are concerned about maintaining purchasing power in times of rising inflation. We formulate investment objectives in terms of real wealth, assuming investors derive utility from the number of goods they can buy with their monetary wealth. We derive closed-form solutions for the portfolio choice problem of constant relative risk averse investors, under the assumption that inflation rates are mean-reverting. We consider alternative specifications for the inflation compensation offered by the available assets, in order to study the effect on portfolio choice and welfare. Moreover, we study the added value of inflationindexed bonds for the investor in our real framework.
\end{abstract}

Keywords: inflation-protection, intertemporal hedging demand, optimal asset allocation, predictability.

\section{JEL Classifications Codes: G11.}

\footnotetext{
${ }^{*}$ Corresponding author: Erasmus University Rotterdam, Econometric Institute, P.O. Box 1738, 3000 DR Rotterdam, The Netherlands. Tel.nr.: +31-104082388, Fax.nr.: +31-104089162, Email: kouwenberg@few.eur.nl
} 


\section{Introduction}

The main purpose of saving is to transfer purchasing power from today to the future. Moreover, through the investment in risky assets investors can participate in the real growth of the economy, at the cost of some additional risk. Traditional consumption-investment models make a tradeoff between the potential gains and losses of investments by considering the overall utility of monetary wealth in dollar terms. Due to this nominal measurement of wealth, the standard models ignore the influence of inflation on the purchasing power of the investor and consequently abstract from the true purpose of saving and investment.

In this paper we formulate investment objectives in terms of real wealth, as we assume that investors derive utility from the number of goods that they can buy with their monetary wealth. We derive closed-form solutions for the portfolio choice problem of constant relative risk averse investors, under the assumption that the inflation rate follows a mean-reverting process. The real-investment framework allows us to address the following important issues, which have not been studied systematically yet:

1. What is the impact of partial inflation-compensation in the drift rate of the asset returns on optimal portfolio choice in a real investment model with mean-reverting inflation?

2. What is the added value of inflation-indexed securities for the investor?

3. How does the optimal portfolio of the investor change if he can additionally invest in inflation-indexed bonds, given estimated coefficients from a recent sample of US asset returns and inflation rates?

We start the analysis by deriving closed-form solutions for the real investment model. Due to the assumption of a mean-reverting process, the optimal portfolio problem includes the inflation rate as an additional state variable. Based on the general model of Merton (1971) we would expect that the portfolio composition changes as a function of the inflation rate. However, if none of the assets has an inflation premium in the drift rate then our closed-form for the optimal asset weights does not depend on the inflation rate.

We demonstrate that this result is caused by the absence of substitution effects: none of the assets has a comparative advantage to hedge against inflation. A change of the inflation rate does affect expected wealth. However wealth effects do not influence the portfolio composition of a constant relative risk averse investor. We also study an economy where assets do contain a (partial) hedge against inflation in the drift rate. In this economy optimal portfolio choice explicitly depends on the inflation rate, due to substitution effects. Furthermore, we show that the investor has a larger demand for the inflation-hedge portfolio for longer investment horizons.

Given the recent period of stable and low inflation rates, one could argue that the additional impact of inflation-uncertainty can be safely ignored in asset-allocation models. However, in the 1960s and 1970s high inflation rates resulted in huge transfers of wealth from the holders of long term bonds to the issuers. The value of a traditional bond erodes when inflation increases, due to the fixed nominal coupons and principal. The danger of eroding bond prices specially affects 
investors with a long investment horizon, including the large group of young workers saving for retirement.

The US Treasury has acknowledged the potential clientele for long term inflation-protection and issues a wide variety of inflation-indexed securities since January 1997 . The principal of these inflation-indexed securities is linked to the value of the CPI, so the payment at maturity is fully protected against inflation. Moreover, the coupon payments are made at a fixed rate relative to the CPI-adjusted principal. Similar inflation-indexed securities are available in the U.K., Canada, Australia, New Zealand, Sweden and Israel. On the Chicago Board of Trade futures and options are even traded with US inflation-indexed bonds as underlying value.

It is therefore surprising that the investment problem with inflation-indexed securities has received little attention in the literature, except for simple mean-variance models (e.g. Bodie 1990 and McFall Lamm 1998). To gain protection against the risk of inflation, the holder of an indexed bond gives up a portion of the interest rate paid on conventional bonds. For example, 30-year conventional bonds issued in 1999 offered an interest rate of $6.125 \%$, while 30-year inflation-indexed bonds issued in 1999 offered 3.875\%. Given our closed-form solution for the real investment model, we can consider the added value of inflation-indexed bonds for investors. We derive the yield that makes the constant relative risk averse investor indifferent between investing all his wealth in traditional assets or additionally holding inflation-indexed bonds.

We conclude that the inflation rate and the risk aversion coefficient of the investor are the most important factors for the minimal required return on inflation-linked bonds. Investors with higher risk aversion accept a lower return on inflation-linked bonds, due to their higher demand for hedging. Regardless of risk aversion, an increase of the inflation rate with $1 \%$ tends to decrease the desired return on inflation-indexed bonds with $1 \%$. Our model indicates that issuers of inflation-linked bonds are forced to increase real rates of return in times of decreasing inflation, in order to create a market for their product.

We conclude the paper with a numerical section, where we investigate the properties of our closed-form solutions further using a recent dataset of US asset returns and inflation from the period 1985-1999. First, we show that the demand for inflation-hedging is rather small in an economy without substitution effects. Second, if we assume that the money market account provides partial compensation against inflation in the drift rate, then the investor adjusts his portfolio with every small change of inflation. Finally, we illustrate that inflation-linked bonds replace conventional bonds in the portfolio of the investor in times of high inflation.

This paper is organized as follows. Section 2 outlines the general investment model for an investor concerned with maintaining purchasing power. In Section 3 we derive closed-form solutions for the optimal portfolio in an economy without substitution effects, where none of the assets possess an inflation premium. Section 4 presents closed-form solutions when substitution effects are present in the economy. The added value of inflation-indexed bonds is the topic of Section 5. In Section 6 we consider a proper statistical process for inflation, using inflation rates in the period January 1985 to October 1999. We also study the inflation-hedging properties of asset returns in this section. Finally, in Section 7 we investigate our closed-form solutions further with estimated coefficients based on a recent sample of US asset returns and inflation. 


\section{Investment in a Real World}

In this section we introduce the portfolio choice problem for an investor who is concerned about real wealth in an economy with a stochastic inflation rate. We will not specify the process for the inflation rate and the utility function of the investor explicitly yet. Instead we consider the general case in this section and we show that the familiar fund separation result holds for any investor. We start our analysis by defining the stochastic processes of the price inflation and returns in the continuous-time economy. The price level is denoted by $N(t)$ and grows instantaneously with the inflation rate $\pi(t)$, which follows an Ito process:

$$
\begin{aligned}
\frac{d N}{N} & =\pi d t, N(0)=1, \\
d \pi & =\mu_{\pi}(\pi, t) d t+\sigma_{\pi}(\pi, t) d Z_{\pi}
\end{aligned}
$$

where $\mu_{\pi}(\pi, t)$ is the instantaneous drift rate and $\sigma_{\pi}(\pi, t)$ is the instantaneous volatility of the inflation rate.

We assume that the investor trades $I+1$ risky assets continuously in a market without transaction costs. The zero-th asset is a money market account $M(t)$, paying interest at the following stochastic rate:

$$
\begin{aligned}
& \frac{d M}{M}=\mu_{B}(\pi) d t+\sigma_{B} d Z_{B}, \\
& d Z_{B} d Z_{\pi}=\rho_{B \pi}
\end{aligned}
$$

We assume that the prices of the remaining assets $P=\left\{P_{i}(t)\right\}_{i=1}^{I}$ are generated by Brownian motions with a drift rate $\mu_{i}(\pi)$ depending on the inflation $\pi$ and a constant volatility $\sigma_{i}$.

$$
\begin{aligned}
& \frac{d P_{i}}{P_{i}}=\mu_{i}(\pi) d t+\sigma_{i} d Z_{i}, \text { for } \forall i \in\{1 . . I\} \\
& d Z_{i} d Z_{j}=\rho_{i j}, d Z_{i} d Z_{\pi}=\rho_{i \pi}, d Z_{i} d Z_{B}=\rho_{i B}, \text { for } \forall i \in\{1 . . I\}
\end{aligned}
$$

As the drift rates of the asset returns and the money market rate may depend on the inflation rate $\pi$, we explicitly allow for assets to contain inflation compensation. In the next sections we will study the impact of inflation compensation in asset returns on optimal portfolio choice.

In order to meet his investment goals the investor dedicates a fraction $w_{i}(t)$ of his wealth to each asset $i$ at time $t$. Hence, the asset-value $A(t)$ of the investor evolves according to the following stochastic differential equation:

$$
\begin{aligned}
d A & =\sum_{i=1}^{I} w_{i} A \frac{d P_{i}}{P_{i}}+\left(1-\sum_{i=1}^{I} w_{i}\right) A \frac{d M}{M} \\
& =\left(\sum_{i=1}^{I} w_{i}\left(\mu_{i}(\pi)-\mu_{B}(\pi)\right)+\mu_{B}(\pi)\right) A d t+\sum_{i=1}^{I} w_{i} A\left(\sigma_{i} d Z_{i}-\sigma_{B} d Z_{B}\right)+\sigma_{B} A d Z_{B}
\end{aligned}
$$

We assume that the investor is not concerned about the monetary value of his wealth $A(t)$, but about the corresponding number of consumption goods that he can buy at today's prices, i.e. 
$A(t) / N(t)$. Consequently, the investor maximizes a utility function $H(., T)$ over real wealth at the planning horizon $T<\infty$ :

$$
\max _{w} E[H(A(T) / N(T), T)]
$$

where $H(., T)$ is an increasing and strictly concave utility function.

This real investment objective and the assumption of a stochastic inflation rate distinguishes our model from the standard nominal setup in the literature. Since the investor is concerned about real wealth, we apply Ito's Lemma to derive the stochastic process for the real asset-value $X(t)=A(t) / N(t)$ :

$$
d X=\left(\sum_{i=1}^{I} w_{i}\left(\mu_{i}(\pi)-\mu_{B}(\pi)\right)+\mu_{B}(\pi)-\pi\right) X d t+\sum_{i=1}^{I} w_{i} X\left(\sigma_{i} d Z_{i}-\sigma_{B} d Z_{B}\right)+\sigma_{B} X d Z_{B}
$$

We now derive the optimal investment strategy by applying the principle of optimality from the dynamic programming literature. According to this principle an optimal policy should maximize the utility of the investor at any point in time, under each economic circumstance. The value function $J(X, \pi, t)$ represents the optimal utility attainable by the investor at time $t$, given his real wealth $X$ and the inflation rate $\pi(t)$. In order to derive the value function $J$ and the corresponding optimal investment policy $w^{*}$, we now state the Hamilton-Jacobi-Bellman equation (see Øxendal 1998):

$$
\begin{aligned}
0= & \max _{w} \phi^{J}(w ; X, \pi, t) \\
= & J_{t}+\left(w^{\prime}\left(\mu(\pi)-\iota \mu_{B}(\pi)\right)+\mu_{B}(\pi)-\pi\right) X J_{X}+\mu_{\pi} J_{\pi} \\
& +\frac{1}{2} w^{\prime} \widehat{\Omega} w X^{2} J_{X X}+\frac{1}{2} \sigma_{B}^{2} X^{2} J_{X X}+\frac{1}{2} \sigma_{\pi}^{2} J_{\pi \pi} \\
& +w^{\prime}\left(\sigma_{I B}-\iota \sigma_{B}^{2}\right) X^{2} J_{X X}+w^{\prime}\left(\sigma_{I \pi}-\iota \sigma_{B \pi}\right) X J_{X \pi}+\sigma_{B \pi} X J_{X \pi}
\end{aligned}
$$

subject to the boundary condition $J(X(T), \pi(T), T)=H(X(T), T)$, where $\widehat{\Omega}=\left\{\widehat{\sigma}_{i j}\right\}_{i, j=1}^{I}$ denotes the covariance matrix of $\left(\sigma_{i} d Z_{i}-\sigma_{B} d Z_{B}\right), \mu(\pi)=\left\{\mu_{i}(\pi)\right\}_{i=1}^{I}$ is the vector of instantaneous drift rates, $\sigma_{I \pi}=\left\{\sigma_{i} \sigma_{\pi}(\pi, t) \rho_{i \pi}\right\}_{i=1}^{I}$ is the covariance vector between the asset returns and the inflation rate and $\sigma_{I B}=\left\{\sigma_{i} \sigma_{B} \rho_{i B}\right\}_{i=1}^{I}$ is the covariance vector between the asset returns and the money market return.

The first order necessary conditions for optimality of the investment policy are:

$$
0=\phi_{w}^{J}=\left(\mu(\pi)-\iota \mu_{B}(\pi)\right) X J_{X}+\widehat{\Omega} w^{*} X^{2} J_{X X}+\left(\sigma_{I B}-\iota \sigma_{B}^{2}\right) X^{2} J_{X X}+\left(\sigma_{I \pi}-\iota \sigma_{B \pi}\right) X J_{X \pi}
$$

If we solve equation (9) for the vector of optimal asset weights $w^{*}$, then we find:

$$
w^{*}=-\widehat{\Omega}^{-1}\left(\sigma_{I B}-\iota \sigma_{B}^{2}\right)-\widehat{\Omega}^{-1}\left(\mu(\pi)-\iota \mu_{B}(\pi)\right) \frac{J_{X}}{X J_{X X}}-\widehat{\Omega}^{-1}\left(\sigma_{I \pi}-\iota \sigma_{B \pi}\right) \frac{J_{X \pi}}{X J_{X X}}
$$

In equation (10) we can distinguish a market portfolio $\widehat{\Omega}^{-1}\left(\mu(\pi)-\iota \mu_{B}(\pi)\right)$, an inflation-hedge portfolio $\widehat{\Omega}^{-1}\left(\sigma_{I \pi}-\iota \sigma_{B \pi}\right)$ and a portfolio to hedge against random fluctuations of the interest rate $\widehat{\Omega}^{-1}\left(\sigma_{I B}-\iota \sigma_{B}^{2}\right)$. We conclude that the investor holds three separating portfolios of risky 
assets and the money market fund. The allocation to these four funds could vary over time as a function of the real asset value $X(t)$ and the inflation rate $\pi(t)$. A nice property of the fund separation result is its generality: it holds for a wide range of utility functions and return processes. However, the value function $J$ is still unknown and we can not derive explicit optimal decision rules without specifying the model further. In the next sections we will provide closedform solutions for a number of cases that are interesting both from an economic perspective and from a practical perspective.

\section{Investing in the Absence of Inflation Premia}

In the previous section we derived the first order conditions for our general investment problem in real terms and we demonstrated that four fund separation applies. We will now specify the inflation process, the asset price processes and the utility function of the investor in order to obtain closed-from solutions. In this section we investigate an economy without inflation compensation in the asset returns. Later on we will consider optimal investment strategies when some assets do provide protection against inflation.

First we focus on the specification of the continuous-time inflation process. As our dataset of monthly US inflation rates from January 1985 up to October 1999 supports the mean-reversion model (see Section 6), we assume that the inflation rate follows a continuous-time OrnsteinUhlenbeck process:

$$
d \pi=\beta(\bar{\pi}-\pi) d t+\sigma_{\pi} d Z_{\pi}
$$

where $\bar{\pi}$ is the long run mean inflation rate and we additionally assume $0<\beta \leq 1$, so meanreversion holds instead of mean aversion $(\beta<0)$.

In order to specify the asset prices processes completely, we still have to decide about the influence of the inflation rate on the drift rates $\mu_{B}(\pi)$ and $\mu_{i}(\pi)$ for $i=1, \ldots, I$. As discussed in Section 6 the major issue is whether the return of an asset provides full, partial or no protection against inflation? Instead of favoring a particular hypothesis for inflation protection of the assets, which might only hold in one country for a particular period of time, we will study alternative asset return specifications in each of the following sections.

In this section we focus on an economy where none of the assets has an inflation premium. We consider the following asset price processes:

$$
\begin{aligned}
& \frac{d M}{M}=r d t+\sigma_{B} d Z_{B}, \\
& \frac{d P_{i}}{P_{i}}=\mu_{i} d t+\sigma_{i} d Z_{i}, \text { for } \forall i \in\{1 . . I\} .
\end{aligned}
$$

Note that the instantaneous interest rate provided by the money market account in (12) is stochastic. The remaining $I$ asset prices follow a geometric Brownian motion (13) with drift rate $\mu_{i} d t$. All asset returns can be correlated with the inflation rate and the interest rate. 
In this section we explicitly derive the optimal investment strategy for the case of constant relative risk aversion. Consequently, the investor maximizes a power utility function over real wealth at the planning horizon:

$$
\max _{w} E\left[\frac{1}{\alpha}(X(T))^{\alpha}\right]
$$

The first order condition for optimality is the HJB-equation (8) with $\mu_{i}(\pi)=\mu_{i}$ and $\mu_{B}(\pi)=r$ for the value function $J(X(t), \pi(t), t)$, subject to the boundary condition:

$$
J(X(T), \pi(T), T)=\frac{1}{\alpha}(X(T))^{\alpha}
$$

In order to satisfy the optimality conditions, we propose the following functional form for the value function:

$$
\begin{aligned}
& J(X(t), \pi(t), t)=\phi(\pi(t), t) \frac{1}{\alpha} X(t)^{\alpha} \\
& \phi(\pi(t), t)=\exp (A(T-t)+B(T-t) \pi(t))
\end{aligned}
$$

Substituting this proposal into the HJB-equation yields a system of ordinary differential equations for the functions $A(T-t)$ and $B(T-t)$. In Appendix A we solve this system of ordinary differential equations and we derive the following expressions for the functions $A(\tau)$ and $B(\tau)$, where $\tau$ denotes $T-t$ for ease of exposition:

$$
\begin{aligned}
& B(\tau)=\frac{\alpha}{\beta}\left(e^{-\beta \tau}-1\right) \\
& A(\tau)=\left(g-f \frac{\alpha}{\beta}+\frac{1}{2} c\left(\frac{\alpha}{\beta}\right)^{2}\right) \tau+\frac{\alpha}{\beta^{2}}\left(c\left(\frac{\alpha}{\beta}\right)-f\right) e^{-\beta \tau}-\left(\frac{1}{2} \frac{\alpha}{\beta}\right)^{2} \frac{c}{\beta} e^{-2 \beta \tau}+\frac{\alpha}{\beta^{2}}\left(f-\frac{3}{4} \frac{\alpha}{\beta} c\right)
\end{aligned}
$$

where $c, f$ and $g$ are constants defined in Appendix A.

Given the expression for the value function $J(X, \pi, t)$, we can now obtain the corresponding optimal portfolio weights from the fund separation result (10):

$$
w^{*}=-\widehat{\Omega}^{-1}\left(\sigma_{I B}-\iota \sigma_{B}^{2}\right)+\frac{1}{1-\alpha} \widehat{\Omega}^{-1}(\mu-\iota r)+\frac{1}{1-\alpha} \widehat{\Omega}^{-1}\left(\sigma_{I \pi}-\iota \sigma_{B \pi}\right) \frac{\alpha}{\beta}\left(e^{-\beta(T-t)}-1\right)
$$

Note that the investor holds the hedge portfolio $\widehat{\Omega}^{-1}\left(\sigma_{I \pi}-\iota \sigma_{B \pi}\right)$, which depends on the correlation between asset returns and inflation. It is remarkable however that the proportion of wealth invested in this inflation hedge portfolio is independent of the inflation rate:

$$
\frac{\partial w^{*}}{\partial \pi}=0
$$

This result can be explained by the substitution and wealth effect of a change of the inflation rate. Since none of the assets contains an inflation premium, substitution effects are absent in our economy. None of the available assets provides a comparative advantage to hedge dynamically against expected changes of the inflation. Hedging demand could emanate from wealth effects however. As wealth effects are absent for investors with constant relative risk-aversion, our investor does not hedge intertemporally against expected changes of the inflation. 
The proportion invested in the inflation hedge portfolio is independent of the investor's wealth and the inflation rate, but does depend on time and the relative risk aversion coefficient of the investor. Whether the hedge portfolio is held long or short depends on the coefficient of relative risk-aversion $(\alpha)$. Log-investors (i.e. $\alpha=0$ ) represent the watershed between buying and selling: they do not invest in the inflation hedge portfolio. Investors whom are more risk-averse than log-investors $(\alpha<0)$ always hold the inflation hedge portfolio long (hedgers). Investors whom are less risk-averse than log-investors $(0<\alpha<1)$ always hold the inflation hedge portfolio short (speculators).

Similarly we investigate the effect of the investment horizon on the optimal investment strategy:

$$
\frac{\partial w^{*}}{\partial \tau}=\frac{-\alpha}{1-\alpha} \widehat{\Omega}^{-1}\left(\sigma_{I \pi}-\iota \sigma_{B \pi}\right) e^{-\beta \tau}
$$

where $\tau=T-t$. We conclude that investors whom are more risk-averse than log-investors have a larger hedging demand for longer investment horizons. The opposite holds for investors whom are less risk-averse than log-investors.

In order to see the impact of the correlation structure more clearly we consider a two-asset economy, consisting of the money market account and one risky stock. In this case equation (20) reduces to the following expression:

$$
\frac{\partial w_{S}^{*}}{\partial \tau}=\frac{-\alpha}{1-\alpha} \frac{\left(\sigma_{S \pi}-\sigma_{B \pi}\right)}{\left(\sigma_{S}^{2}-2 \sigma_{S B}+\sigma_{B}^{2}\right)} e^{-\beta \tau}=\frac{-\alpha}{1-\alpha} \frac{\sigma_{\pi} \sigma_{S}}{\left(\sigma_{S}^{2}-2 \sigma_{S B}+\sigma_{B}^{2}\right)}\left(\rho_{S \pi}-\rho_{B \pi} \frac{\sigma_{B}}{\sigma_{S}}\right) e^{-\beta \tau}
$$

where $w_{S}^{*}$ is the optimal stock weight, $\sigma_{S}^{2}$ denotes the instantaneous variance of the stock return and $\rho_{S \pi}$ and $\rho_{S B}$ denote the correlation of the stock return with the inflation rate and the money market rate respectively.

Equation (21) shows that an increase of the correlation between stock returns and inflation tends to strengthen the horizon effect. The opposite holds for the correlation of the money market rate with inflation, but this effect will be less strong due to the scaling factor $\sigma_{B} / \sigma_{S}<1$. Whether the horizon effect for the stock weight is positive or negative depends on the sign of the expression $-\alpha\left(\rho_{S \pi}-\rho_{B \pi}\left(\sigma_{B} / \sigma_{S}\right)\right)$. If the investor is more risk-averse than a log-investor $(\alpha<0)$ we conclude that the stock weight decreases (increases) for longer horizons if $\rho_{S \pi}<(>) \rho_{B \pi}\left(\sigma_{B} / \sigma_{S}\right)$ holds.

In contrast to Merton's results for a nominal economy, we find that investors behave non-myopic in our real investment model. Another remarkable result is that the hedging demand does not depend on the inflation rate. This can be explained by the absence of substitution effects in the economy studied in this section. None of the available assets has a comparative advantage to hedge against expected changes of the inflation rate. In the next section we investigate the optimal investment strategy if some assets returns include an inflation premium.

\section{Portfolio Choice in the Presence of Inflation Premia}

In the previous section we assumed that none of the assets provides compensation for inflation. This assumption might be unrealistic however. The results of Section 6 demonstrate that 
short-term interest rates provided a partial hedge against expected inflation during the period 1985-1999. In this section we study an economy where the money market account provides protection against expected inflation, and all the other assets may or may not provide inflation compensation. We consider the following stochastic processes in the economy:

$$
\begin{aligned}
& d \pi=\beta(\bar{\pi}-\pi) d t+\sigma_{\pi} d Z_{\pi}, \\
& \frac{d M}{M}=(\pi+r) d t+\sigma_{B} d Z_{B}, \\
& \frac{d P_{i}}{P_{i}}=\left(\lambda_{i} \pi+\mu_{i}\right) d t+\sigma_{i} d Z_{i}, \text { for } \forall i \in\{1 . . I\} .
\end{aligned}
$$

Note that the expected inflation at time $t+d t$ approximately equals $E\left[\pi_{t+d t}\right] \approx \pi_{t}+\beta\left(\bar{\pi}-\pi_{t}\right) d t$. If the money market account provides compensation for expected inflation then its drift rate at time $t$ equals $\left(E\left[\pi_{t+d t}\right]+r\right) d t \approx\left(\pi_{t}+r\right) d t$, as $d t d t=0$. Consequently, the rate of return on the money market account in (23) is equal to the sum of the instantaneous expected inflation rate $\pi(t) d t$ and the stochastic real rate of return $r d t+\sigma_{B} d Z_{B}$. The other risky assets follow a geometric Brownian motion (24) with drift rate $\left(\lambda_{i} \pi+\mu_{i}\right) d t$. The asset returns can additionally be correlated with the inflation rate and the real interest rate.

The first order conditions of optimality for the value function $J(X(t), \pi(t), t)$ and the investment policy are given by the HJB-equation (8) with $\mu_{i}(\pi)=\lambda_{i} \pi+\mu_{i}$ and $\mu_{B}(\pi)=\pi+r$, subject to the boundary condition:

$$
J(X(T), \pi(T), T)=\frac{1}{\alpha}(X(T))^{\alpha}
$$

We propose the following functional form for the value function:

$$
\begin{aligned}
& J(X(t), \pi(t), t)=\Phi(\pi(t), t) \frac{1}{\alpha}(X(t))^{\alpha} \\
& \Phi(\pi(t), t)=\exp \left(A(T-t)+B(T-t) \pi(t)+\frac{1}{2} C(T-t) \pi(t)^{2}\right)
\end{aligned}
$$

If we substitute the proposal (26) into the HJB-equation (8), then this partial differential equation reduces to a more tractable system of ordinary differential equations for $C(\tau), B(\tau)$ and $A(\tau)$ :

$$
\begin{aligned}
& C^{\prime}(\tau)=a+b C(\tau)+c C(\tau)^{2}, \\
& B^{\prime}(\tau)=d+\frac{1}{2} b B(\tau)+f C(\tau)+c B(\tau) C(\tau), \\
& A^{\prime}(\tau)=g+f B(\tau)+\frac{1}{2} h C(\tau)+\frac{1}{2} c B(\tau)^{2},
\end{aligned}
$$

where $a, b, c, d, f, g$ and $h$ are constants, defined in Appendix A.

The system of ordinary differential equations (27) can be classified as a system of Ricatti equations and can be solved recursively. The mathematical form of the solution depends on the discriminant $q$ of the Riccati equation for $C(\tau)$ :

$$
q=b^{2}-4 a c=4 \beta^{2}+4 \frac{\alpha^{2}}{(\alpha-1)^{2}}\left(H_{14}^{2}-H_{11} H_{44}\right)+4 \frac{\alpha}{(\alpha-1)}\left(\sigma_{\pi}^{2} H_{11}-2 \beta H_{14}\right)
$$


where $H_{11}=(\lambda-\iota 1)^{\prime} \widehat{\Omega}^{-1}(\lambda-\iota 1), H_{14}=(\lambda-\iota 1)^{\prime} \widehat{\Omega}^{-1}\left(\sigma_{I \pi}-\iota \sigma_{B \pi}\right)$ and $H_{44}=\left(\sigma_{I \pi}-\right.$ $\left.\iota \sigma_{B \pi}\right)^{\prime} \widehat{\Omega}^{-1}\left(\sigma_{I \pi}-\iota \sigma_{B \pi}\right)$.

The solution for $C(\tau)$ and $B(\tau)$ in the case $q>0$ is:

$$
\begin{aligned}
C(\tau)= & \frac{2 a\left(1-e^{-\eta \tau}\right)}{2 \eta-(b+\eta)\left(1-e^{-\eta \tau}\right)} \\
B(\tau)= & {\left[\left(-2 d(b+\eta)-\frac{f}{c}(b+\eta)^{2}+2 \frac{f}{c} b(b+\eta)\right)\left(e^{-\eta \tau / 2}-1\right)\right.} \\
& \left.-\left(\frac{f}{c}(\eta-b)(\eta+b)+2 d(b-\eta)\right)\left(e^{\eta \tau / 2}-1\right)\right] \frac{e^{-\eta \tau / 2}}{\eta\left[2 \eta-(b+\eta)\left(1-e^{-\eta \tau}\right)\right]}
\end{aligned}
$$

where $\eta=\sqrt{b^{2}-4 a c}$.

A remarkable feature of the solution is that the value function might reach infinity in finite time. Kim and Omberg (1996) refer to these cases as nirvana solutions. For $q>0$ nirvana solutions only occur if condition (31) is satisfied. For $q<0$ every solution is a nirvana solution, while in the border case $q=0$ additionally $b>0$ has to hold (Appendix A contains a full derivation of all possible solutions).

$$
a c>0, b>0 \text { and } T>\frac{1}{\eta} \ln \left(\frac{b+\eta}{b-\eta}\right)
$$

Investment strategies that can reach infinite utility in finite time might cast a doubt on continuoustime models with perfect market assumptions. In practice market imperfections like transaction costs and borrowing constraints clearly prevent unbounded solutions. An important question is whether nirvana solutions actually occur for reasonable parameter values?

In Appendix B we show that in a two-asset economy $q>0$ always holds and nirvana solutions never occur. For economies with more than 2 assets the covariance matrix complicates the analysis and we can not provide an explicit proof. However, in Section 7 we demonstrate that for the estimated parameters of the US dataset $q>0$ holds and that nirvana can not be reached. From now, we will therefore assume $q>0$ and ignore the possibility of nirvana solutions.

Given the expression for the value function $J(X, \pi, t)$ in the case $q>0$, we can now derive the optimal risky asset weights from the fund separation equation (10). Note that the solution (32) simplifies considerably if all assets contain an exact unit inflation premium $(\lambda=\iota)$, and therefore we will analyze that case separately later.

$$
\begin{aligned}
w^{*}(\pi(t), t)= & -\widehat{\Omega}^{-1}\left(\sigma_{I B}-\iota \sigma_{B}^{2}\right)+\widehat{\Omega}^{-1} \frac{((\lambda-\iota 1) \pi+\mu-\iota r)}{1-\alpha} \\
& +\frac{\widehat{\Omega}^{-1}\left(\sigma_{I \pi}-\iota \sigma_{B \pi}\right)}{1-\alpha}[B(T-t)+C(T-t) \pi(t)]
\end{aligned}
$$

First we consider the case $\lambda \neq \iota$. Note that the demand for the inflation hedge portfolio $\widehat{\Omega}^{-1}\left(\sigma_{I B}-\iota \sigma_{B}^{2}\right)$ depends on time $t$ and on the inflation rate $\pi(t)$. Moreover, the fraction of wealth invested in the market portfolio $\widehat{\Omega}^{-1}(\mu+\lambda \pi-\iota(r+\pi))$ is non-myopic, since the inflation rate affects the drift rates of the asset returns. We conclude that the inflation rate influences 
the portfolio composition, as a result of the substitution effect between assets with and without an inflation premium. The sign of the relation between the inflation rate and portfolio weights is not clear in general: we refer to Section 7 for a numerical investigation.

We now focus the special case where all assets returns have an exact unit inflation premium, i.e. $\lambda_{i}=1$ for $\forall i \in\{1,2, \ldots, I\}$. We prove in Appendix A that $C(t)=B(t)=0$ holds in this case and consequently the value function reduces to a function $J(X(t), t)$ of real wealth and time only. As a result, the optimal investment strategy (32) simplifies to:

$$
w^{*}=-\widehat{\Omega}^{-1}\left(\sigma_{I B}-\iota \sigma_{B}^{2}\right)+\widehat{\Omega}^{-1} \frac{(\mu-\iota r)}{1-\alpha}
$$

Hence, under the additional assumption $\lambda=\iota$ the inflation rate has no influence on the optimal portfolio choice of an investor with constant relative risk aversion.

If we additionally assume $\sigma_{B}=0$, then (33) becomes equivalent to the optimal investment strategy of Merton (1969). We conclude that the myopic solution of Merton (1969) is only a special case in our real economy with mean-reverting inflation. Finally, we would like to point out that if $\sigma_{B}=0$ holds, then the money market provides the instantaneous return $(r+\pi(t)) d t$ and consequently becomes a complete hedge against inflation. It could be interpreted as an inflation-indexed bond that can be returned to the issuer at any point in time. We will study the demand for this inflation-indexed security more closely in the next section.

\section{Inflation-Indexed Securities}

In the previous section we discussed portfolio choice in an economy where some assets provide partial inflation-compensation. Recently securities have been issued in the US that guarantee full protection against inflation, so called inflation-indexed securities. Guaranteed full protection against changes of the CPI is a feature that traditional securities lack. As this additional feature could be valuable for investors, the inflation-indexed securities might yield a lower real return. We study the value of inflation-protection by deriving the lower bound of real returns at which the investor is willing to buy inflation-indexed securities.

We consider the following economy

$$
\begin{aligned}
& d \pi=\beta(\bar{\pi}-\pi) d t+\sigma_{\pi} d Z_{\pi}, \\
& \frac{d L}{L}=\left(\pi+r^{*}\right) d t, \\
& \frac{d M}{M}=(\pi+r) d t+\sigma_{B} d Z_{B}, \\
& \frac{d P_{i}}{P_{i}}=\left(\lambda_{i} \pi+\mu_{i}\right) d t+\sigma_{i} d Z_{i}, \text { for } \forall i \in\{1 . . I\},
\end{aligned}
$$

Compared to the previous section the investor can now additionally invest in an inflation-indexed security with price $L(t)$. It can be interpreted as a puttable inflation-indexed bond with infinite 
maturity. Effectively the owner has the right to sell the security to the issuer at any point in time, in return for the CPI-linked principal $N(t)$ and the accumulated interest payments at the constant yield $r^{*}$.

The optimal real investment problem with inflation-indexed securities can be solved analogously to the problem of the previous section. We introduce the inflation-indexed bond as 'riskless' security (i.e. substitute $r=r^{*}$ and $\sigma_{B}=0$ in all formulas) and we consider the money market account as risky asset $I+1$, with $\mu_{I+1}=r, \sigma_{I+1}=\sigma_{B}$ and $d Z_{I+1}=d Z_{B}$. As a result we find the following optimal investment strategy for a constant relative risk-averse investor, in the normal case $q>0$ :

$$
w^{*}(\pi(t), t)=\frac{1}{1-\alpha} \widetilde{\Omega}^{-1}\left(\mu+\lambda \pi-\iota\left(r^{*}+\pi\right)\right)+\frac{1}{1-\alpha} \widetilde{\Omega}^{-1} \sigma_{I \pi}[B(T-t)+C(T-t) \pi]
$$

where $\widetilde{\Omega}$ is the covariance matrix of $\left\{d Z_{i}\right\}_{i=1}^{I+1}$. Nirvana solutions could exist hypothetically (we refer to Appendix A for the exact conditions), but as indicated in Section 7 the occurrence of these solutions is very unlikely for reasonable parameter values.

We will now focus on the added value of the inflation-protected security for the investor. For this purpose we determine the value $\underline{r}^{*}$ at which the investor becomes indifferent between investing and not investing in the inflation-indexed bond. The fraction of real wealth invested in the inflation-indexed bond is given by:

$$
\begin{aligned}
w_{0}^{*} & =1-\iota^{\prime} w^{*} \\
& =1+\frac{1}{1-\alpha} \iota^{\prime} \widetilde{\Omega}^{-1}\left(\mu+\lambda \pi-\iota\left(r^{*}+\pi\right)\right)+\frac{1}{1-\alpha} \iota^{\prime} \widetilde{\Omega}^{-1} \sigma_{I \pi}[B(T-t)+C(T-t) \pi]
\end{aligned}
$$

Hence, setting $w_{0}^{*}=0$, we can solve for the lower bound $\underline{r}^{*}$ of the real rates of return at which the investor is willing to buy the inflation-indexed bond ${ }^{1}$ :

$$
\underline{r}^{*}=\frac{A_{1}-(1-\alpha)+A_{2} B_{0}(\tau)+\left[A_{3}-A_{0}+A_{2} C(\tau)\right] \pi}{A_{0}+\frac{\alpha}{1-\alpha} A_{2}^{2} B_{2}(\tau)-\frac{\alpha}{1-\alpha} A_{2}\left(A_{3}-A_{0}\right) B_{1}(\tau)}
$$

where $\tau=T-t, A_{0}=\iota^{\prime} \widetilde{\Omega}^{-1} \iota, A_{1}=\iota^{\prime} \widetilde{\Omega}^{-1} \mu, A_{2}=\iota^{\prime} \widetilde{\Omega}^{-1} \sigma_{I \pi}, A_{3}=\iota^{\prime} \widetilde{\Omega}^{-1} \lambda$ and $B_{1}(\tau)=$ $2 \frac{b}{\eta} G(\tau)-\frac{1}{a} C(\tau)$ and $B_{2}(\tau)=\frac{4 a}{\eta} G(\tau)$, where we introduced the function

$$
G(\tau)=\frac{\left(1-e^{-\eta \tau / 2}\right)^{2}}{\left[2 \eta-(b+\eta)\left(1-e^{-\eta \tau}\right)\right]}
$$

Note that $\underline{r}^{*}$ can also be interpreted as a break-even yield: the inflation-indexed bond is a profitable investment for the investor as long as $r^{*}$ exceeds $\underline{r}^{*}$. We would like to know the effect of a change of the inflation rate on the break-even return. Unfortunately this relation is not clear-cut, due to the unknown sign of the term in front of $\pi$ in (41). In order to enhance tractability we therefore consider a two-asset economy with an inflation-indexed bond and a

\footnotetext{
${ }^{1}$ Note that the function $B(T-t)$ depends on the yield of the inflation-indexed bond $r^{*}$. Without a closed-form solution for the portfolio weights we could not solve for the break-even yield $\underline{r}^{*}$, due to the possible dependence of the value function on $r^{*}$.
} 
stock, where the stock return is uncorrelated with the inflation $\left(A_{2}=0\right)$. In this case (41) reduces to:

$$
\underline{r}^{*}=\mu_{S}-(1-\alpha) \sigma_{S}^{2}-\left(1-\lambda_{S}\right) \pi
$$

where $\mu_{S}$ and $\sigma_{S}$ denote the mean and the volatilty of the stock return respectively and $\lambda_{S}$ is the inflation-compensation parameter of the stock returns.

We conclude that an increase of inflation will reduce the break-even return on the inflationindexed bond, if the stock provides less than unit inflation-compensation $\left(\lambda_{S}<1\right)$. In particular, a percentage increase in inflation, reduces the break-even return with $\left(1-\lambda_{S}\right)$ percent. Another conclusion is that investors with a higher risk aversion coefficient $\alpha$ accept a lower break-even return. Higher risk aversion leads to an increased demand for inflation hedging. Furthermore, an increase of the expected stock return $\mu_{S}$ and a decrease of the volatilty $\sigma_{S}^{2}$ both increase the break-even return, due to substitution effects. In Section 7 we will study the break-even yield numerically for the multiple-asset case, based on actual US data. In the next section we consider US inflation rates and study the inflation-hedging properties of conventional asset returns in the period January 1985 to October 1999.

\section{Inflation and Asset Returns}

In the previous sections we derived closed-form solutions for the optimal portfolio choice in the real investment model with mean-reverting inflation rates. In the next section we investigate these closed-form solutions using US data on asset returns and inflation from the period January 1985 to October 1999. In this section we first consider the statistical properties of inflation, and show that our dataset supports the mean-reverting model for inflation. In the previous sections we considered different economies where assets may or may not possess a partial inflation-hedge. In this section we study these inflation-hedging properties for actual asset returns over our sample period.

Given the recent period of stable and low inflation rates, one might argue that the impact of inflation-uncertainty can safely be ignored in asset-allocation models. However, the 1960s and 1970s marked a period of high inflation rates in many developed countries. Figure 1 shows the monthly US inflation rate based on the seasonally adjusted Consumer Price Index, for the period January 1952 to October 1999. Until approximately 1967 inflation is stable at a low level. Then, the financing of the Vietnam War has a clear impact on prices. In 1973 the oil crisis causes inflation rates to double. Halfway 1981 the period of high inflation ends.

It is clear that inflation rates are neither negligible nor constant and should therefore be taken into account in consumption-investment models. This issue has become even more urgent lately, as the US Treasury regularly issues inflation-indexed bonds since 1997. Apparently there is a demand for assets that provide protection against inflation. In this paper we formulated investment objectives in terms of real wealth, as we assumed that investors derive utility from the number of goods represented by monetary wealth. We assumed that inflation rates are 
mean-reverting and considered alternative specifications for the inflation compensation of the available assets.

In this section we verify these assumptions by considering actual US data in the period January 1985 to October 1999. The two main issues we address in this section are:

1. What is a proper statistical model for describing stochastic inflation rates?

2. To what extent do asset returns provide protection against inflation?

We start with the first issue, the statistical process of the inflation. It is widely accepted that the inflation rate is not independently distributed through time, but displays predictable patterns. However, there is an ongoing debate about whether this predictability should be modeled with a stationary mean-reverting process or a non-stationary long memory timeseries model. Hassler and Wolters (1995) provide international evidence of long memory in inflation rates. Bos, Franses and Ooms (1999) on the other hand argue that the long run memory property of inflation series might occur due to underlying level shifts, which can be interpreted as exogenous shocks.

Exogenous shocks can completely change the range of observed inflation rates: consider for example the impact of the oil crisis and the Vietnam war on the US inflation rates in Figure 1. Clearly, it is very hard to predict when the next structural break in the inflation series might occur. For financial planning purposes it therefore seems more reasonable to stick to the stable mean reversion property of inflation rates. As our dataset of asset returns starts in 1985, we focus on the inflation rates in the period January 1985 to October 1999.

We investigate whether monthly US inflation rates support the mean-reverting model during this period, by estimating the regression model (44).

$$
\begin{aligned}
\Delta \pi_{t} & =\alpha_{0}+\alpha_{1} \pi_{t-1}+\epsilon_{t}, \epsilon_{t} \sim \operatorname{IID}\left(0, \sigma_{\epsilon}\right) \\
& =\beta\left(\mu_{\pi}-\pi_{t-1}\right)+\epsilon_{t},
\end{aligned}
$$

where $\pi_{t}$ is the inflation in month $t=1, \ldots, T, \Delta \pi_{t}=\pi_{t}-\pi_{t-1}, \alpha_{0}$ and $\alpha_{1}$ are coefficients of the regression, $\epsilon_{t}$ for $t=1, . ., T$ are identically and independently distributed (IID) error terms, $0<\beta \leq 1$ and $\mu_{\pi}$ are parameters for the speed of mean-reversion and the long run mean inflation rate respectively.

If the parameter $\beta$ is positive and $\beta \leq 1$ mean-reversion holds and the inflation rate will be pulled back to the long run mean $\mu_{\pi}$. In the case $\beta=0$ the inflation rate follows a random walk. If $\beta<0$ holds, the inflation rate will move away from the mean. Consequently, the nullhypothesis of no mean-reversion is equivalent to $-1 \leq \alpha_{1}<0$ in the regression model (44). The results of the regression are summarized in Table 1. The estimated value of $\alpha_{1}$ is significantly negative and above -1 , so we find strong evidence for mean reversion.

We will now turn to the second issue: to what extend do asset returns provide compensation against inflation? The traditional view among economists is that risky assets should provide a hedge against expected inflation. The Fisher (1930) hypothesis states that the expected nominal 
return is equal to the sum of the expected real return and the expected inflation rate:

$$
E\left[r_{i t}\right]=E\left[r_{i t}^{*}\right]+E\left[\pi_{t}\right]
$$

where $r_{i t}$ is the nominal return on asset $i$ in period $t, r_{i t}^{*}$ is the real return on asset $i$ and $\pi_{t}$ is the inflation rate.

Compelling empirical evidence demonstrates, however, that stock returns in the United States are either unrelated or negatively related to inflation, inconsistent with the Fisher hypothesis (Bodie 1976, Fama and Schwert 1977, and Geske and Roll 1983). Similar results hold for bond returns (Fama 1981, and Fama and Gibbons 1982). More recent empirical studies by Barnes et al. (1999) confirm that equity returns and interest-rates are poor hedges for inflation in most Western countries. Real estate might have good inflation-hedging properties (Fama and Schwert 1977, Ibbotson and Siegel 1984, and Goetzmann and Ibbotson 1990), however these results could depend on the particular index chosen to represent real estate.

For illustrative purposes we will now shortly study the inflation hedging properties of several broad asset classes in the US. We gathered the 1-month financial commercial paper rate and the monthly return on 1-year Treasury Bills from the Federal Reserve Economic Database. We use the Salomon Brothers Bond Index for a maturity of 10 years and longer to represent long term bond investments. Furthermore, we use monthly returns on the S\&P500 index for the stock market. The choice for a proper real estate index is less straightforward. Most unsecuritized real estate data, such as residential real estate used in the study of Fama and Schwert (1977), are subject to appraisal-smoothed biases (see Geltner 1991). We use a total return index on real estate investment trusts (REITs) as a proxy for investing in US real estate, provided by the National Association of Real Estate Investment Trusts.

Table 2 summarizes the average return and volatility for the different asset classes. It is remarkable that the REIT returns are dominated by the returns on bonds and stocks in terms of mean-variance efficiency. Note that the REIT and S\&P500 returns are quite volatile and moreover display fat tails. To some extent these stylized facts can be attributed to the October 1987 crash, which is included in our dataset. In order to investigate the inflation-hedging properties of the asset returns, we will examine the correlations with the monthly inflation rate. Table 3 supports the conclusions from the literature on the negative relation between stock returns and inflation. Remarkably, the equity REIT returns are also negatively related to inflation during the sample period January 1985-October 1999. Commercial paper rate and 1-year Treasury bills on the other hand are strongly positively correlated with inflation.

In order to provide more insight into inflation-compensation we test the Fisher (1930) hypothesis with a regression of asset returns on inflation, displayed in (45). We use the contemporaneous rate of inflation as a proxy for expected inflation, relying on the rational expectations hypothesis (see Gultekin 1983). We have also tested the Fisher hypothesis with the 3-month T-bill rate as a proxy for expected inflation and found comparable results.

$$
r_{i t}=\gamma_{0}+\gamma_{1} \pi_{t}+\epsilon_{t}, \epsilon_{t} \sim \operatorname{IID}\left(0, \sigma_{\epsilon}\right)
$$

where $r_{i t}$ is the monthly return on asset $i, \pi_{t}$ denotes the monthly inflation rate, $\gamma_{0}$ and $\gamma_{1}$ are 
the coefficients of the regression, $\epsilon_{t}$ for $t=1, . ., T$ are identically and independently distributed (IID) error terms

The results of regression (45) are summarized in Table 4. We confirm the findings in the literature for bonds and stocks: the returns of bonds and stocks are negatively related or at best unrelated to inflation rates. Furthermore, we also find that there is no evidence of a positive relation between REIT returns and inflation. Apparently real estate is a poor inflation-hedge, if we use an index based on actual market values. On the other hand, the commercial paper rate and the return on 1-year T-bills are positively related with inflation, and do provide a partial hedge against inflation.

We conclude from this section that inflation rates follow a mean-reverting process in the recent period 1985-1999. We confirmed that the inflation hedging properties of asset returns are weak, although short-term interest rates seem to provide a partial compensation for expected inflation. In the next section we use our dataset of US asset returns and inflation to investigate our closedform solutions further.

\section{Numerical Examples for the Period 1985-1999}

In the previous sections we derived closed-form solutions for the real investment problem with mean-reverting inflation rates. In some cases ambiguity remained however about the sign and magnitude of coefficients and therefore some interesting economic questions could not be fully addressed. In this section we will further investigate the closed-form solutions by performing some explicit calculations using the US data on asset returns and inflation from the period January 1985 to October 1999 discussed in the previous section. In particular we address the following questions:

1. Do nirvana solutions actually occur for reasonable parameter values?

2. How much does the optimal portfolio in an economy without substitution effects deviate from the myopic portfolio of Merton (1969)?

3. What are the implications for the optimal portfolio if some assets provide partial protection against expected inflation?

4. How much return is the holder of an indexed-linked bond willing to give up in order to gain protection against inflation-risk?

\subsection{The Likelihood of Nirvana}

We first turn our attention to the occurrence of nirvana solutions. As we discussed in the previous sections a peculiar feature of the optimal solutions is that the value function might reach infinity in finite time for certain parameter combinations. Kim and Omberg (1996) refer to such solutions as nirvana solutions. In Appendix A we derive the following necessary conditions 
for the occurrence of nirvana solutions in the case $q>0$

$$
a c>0, b>0 \text { and } T>\frac{1}{\eta} \ln \left(\frac{b+\eta}{b-\eta}\right)
$$

In Appendix B we prove that in a two-asset economy nirvana solutions can never occur and that $q>0$ always holds. With more than two assets the covariance matrix of the returns complicates the analysis and hence we can not provide an explicit proof. In order to provide more insight we now investigate whether nirvana solutions occur for the estimated parameters of our US dataset of asset returns. We consider an investor with a horizon of 20 years and coefficient of relative risk-aversion $\alpha=-0.5$. For a description of the securities, summary statistics and regression results we refer to Section 6 .

First, we consider an economy with an inflation-indexed bond, where none of the other assets possesses an inflation premium. We assume that the interest rate on the inflation-indexed bond equals $3.5 \%$. This yield is higher than the average real return on short-term T-bills, but is consistent with current ${ }^{2}$ market rates on indexed-linked Treasuries. Additionally, the investor may invest in the Salomon Brothers Bond Index with maturity over 10 years, the S\&P500, 1-year T-bills, and Equity REITs. The estimated values of the parameters $q, a c$, and $b$ are summarized in the first panel of Table 5. We conclude that $q>0, a c<0$ and $b<0$ hold, so nirvana solutions are out of the question for the sample of asset returns from the period 1985-1999.

Second, we consider an economy without inflation-indexed bonds, where short-term interestrates provide a partial hedge against inflation. In Section 6 we regressed asset returns on inflation, yielding estimated coefficients for commercial paper and 1-year T-bills of $\gamma_{1}=0.22$ and $\gamma_{1}=0.19$ respectively. Given these parameters for the inflation premia, the second panel of Table 5 summarizes the results for different investment opportunities. Again we find that $q>0$, $a c<0$ and $b<0$ always hold and consequently nirvana solutions are impossible.

Note that the values of $a, b$, and $c$ depend on the coefficient of relative-risk aversion $\alpha$, which we arbitrarily fixed at -0.5 . However, additional computations show that nirvana solutions do not occur for any value of $\alpha$. For $\alpha<0, a c<0$ holds in our dataset; hence nirvana is impossible. For $0 \leq \alpha<1, a c$ may become positive, however in this case $b<0$; again we conclude that nirvana solutions can not occur. Given our proof for the two-asset case (Appendix B) and our numerical experiments with actual data, we conclude that nirvana solution are very unlikely for common parameter values.

\subsection{Horizon-Effects in an Economy without Inflation-Premia}

We now turn to the second question. How much does the optimal portfolio in an economy without substitution effects deviate from the myopic portfolio of Merton (1969)? The difference between the non-myopic investment strategy (19) of Section 3 and Merton's myopic portfolio is

\footnotetext{
${ }^{2}$ December 1999
} 
represented by the following time-dependent investment in the inflation-hedge portfolio:

$$
\frac{1}{1-\alpha} \widehat{\Omega}^{-1}\left(\sigma_{I \pi}-\iota \sigma_{B \pi}\right) \frac{\alpha}{\beta}\left(e^{-\beta(T-t)}-1\right)
$$

We would like to know the magnitude of expression (46) for our dataset of monthly US asset returns.

Before we calculate the investment strategies, we would like to stress that the returns of EREIT's are dominated in terms of mean-variance by bonds and the S\&P500 and negatively correlated with inflation (see Section 6). Therefore we ignore these securities as an investment opportunity. Furthermore, the characteristics of 1-year T-bills and commercial paper are very similar. Including both assets might result in extreme investment strategies exploiting the small differences in these assets. We will now demonstrate with an example that these effects are not limited to the continuous-time framework, but may occur in any portfolio optimization model ignoring transaction costs and parameter-uncertainty.

For illustrative purposes we consider a simple mean-variance optimization model where the investor can invest in commercial paper, 1-year T-bills, the Salomon Brothers Bond Index and the S\&P500. An investor desiring an expected return equal to $15 \%$ annually, would take a long position of $1486.7 \%$ in commercial paper, short T-bills with $-1433.7 \%$ and invest $24.1 \%$ in bonds and $22.9 \%$ in stocks. Clearly such extreme policies are caused by the similar meanvariance properties of commercial paper and T-bills. Therefore, from now on we will ignore 1-year T-bills as an investment opportunity.

Given 1-month commercial paper (serving as the money market account), the Salomon Brothers Bond Index and the S\&P500 as investment opportunities, we now quantify the inflation-hedge portfolio (46) in an economy without substitution effects. Again we consider an investor with a horizon of 20 years and coefficient of relative risk-aversion $\alpha=-0.5$. We apply the estimated parameter values of Section 6 as coefficients for the continuous-time model. As a result, we find that the estimated components of the vector $\widehat{\Omega}^{-1}\left(\sigma_{I \pi}-\iota \sigma_{B \pi}\right)$ are equal to 0.0027 for bonds and -0.0082 for stocks respectively.

The inflation-hedge portfolio (46) mostly consists of short term interest-rate securities, due to their positive correlation with inflation. We conclude that, compared to Merton's myopic portfolio, our investor transfers wealth from stocks (S\&P 500) to commercial paper and bonds. Figure 2 shows the fraction invested in the S\&P500 as time evolves. Clearly, a power-utility investor who takes mean-reverting inflation into account no longer behaves myopic, as we discussed in Section 3. We conclude, however, that the actual magnitude of the time-effect is rather small for the US data studied here.

\subsection{The Impact of Substitution Effects on Portfolio Choice}

In Section 4 we studied an economy where some assets provide a partial hedge against inflation. We found that in an economy where the asset returns contain inflation premia the optimal portfolio for a constant relative risk-averse investor depends both on time and inflation rates. 
Due to substitution effects between assets with and without explicit inflation premia the investor will adjust the portfolio composition if the inflation rate changes. We will now quantify these substitution effects for our historical dataset of US asset returns from the period 1985-1999.

The investor can invest in the same assets as before: 1-month commercial paper, the Salomon Brothers Bond Index and the S\&P500. The horizon of the investor is 20 years and his coefficient of relative risk-aversion is $\alpha=-0.5$. In this economy with substitution effects, the inflationpremium of commercial paper is given by $\gamma_{1}=0.22$ (see Section 6 ). In order to calculate the investor's optimal portfolio completely, we additionally require a path of realized inflation rates.

Rather than considering simulated scenarios for the inflation rates, we calculate the optimal portfolios during the historical period January 1985-October 1999. In Figure 3 we plot the percentage that our investor would have invested in the S\&P500 during this particular period, with monthly rebalancing. As a benchmark, we also display the fraction S\&P500 for the economy without substitution effects (no inflation premia), which remains nearly constant around $65.5 \%$. We conclude from Figure 3 that in an economy with substitution effects the weight of the S\&P500 is adjusted as the inflation rate changes.

Generally, the percentage invested in the S\&P500 fluctuates around $64.5 \%$ with an approximate bandwidth of only $2 \%$. Some obvious outliers occur around March and May 1986, when inflation rates were negative, and around February and September 1990, when inflation rates ran in double figures (annualized). The impact of such extreme movements of the inflation rate on the optimal portfolio is, however, relatively small. On average the investor dedicates less to stocks when substitution effects are present, as funds are transferred to the asset with partial inflation-compensation.

We conclude that the assumption of compensation for inflation in the drift rate of the assets only has a slight impact on the composition of the portfolio. Brennan, Schwartz and Lagnado (1997) show numerically that return predictability can have a drastic influence on portfolio choice. For an extremely prudent investor with relative risk-aversion coefficient equal to -5 and with shortselling constraints, they find that the optimal fraction invested in stocks bounces up and down between $0 \%$ and $100 \%$. In reality excessive rebalancing is costly due to transaction costs. From Figure 3 we conclude that the impact of inflation predictability on the optimal portfolio weights is relatively small.

\subsection{The Demand for Inflation-Indexed Bonds}

Finally, in Table 6 we display the demand for inflation-indexed bonds for an investor with a horizon of 20 years and coefficient of relative risk-aversion $\alpha=-1.0$. The additional availability of an inflation-indexed bond induces the investor to hedge intertemporally against inflation. Clearly, a higher inflation rate increases the demand for inflation-indexed bonds, while conventional bonds and stocks are driven out of the optimal portfolio. When inflation changes from $0 \%$ to $10 \%$ the exposure to stocks is halved, while the exposure to conventional bonds is reduced 7-fold. Simultaneously the investor increases the proportion invested in the inflation-indexed bond from $0 \%$ to $65 \%-70 \%$. We conclude that inflation-indexed bonds drive traditional bonds 
out of the portfolio in times of high inflation.

The remaining question we investigate in this section is how valuable the inflation-indexed bond is to our investor. To gain protection against the risk of inflation, the holder of an indexed bond gives up a portion of the interest rate paid on conventional bonds. For example, 30-year conventional bonds issued in 1999 offered an interest rate of $6.125 \%$, while indexed 30-year bonds issued in 1999 offered 3.875\%. In Section 5 we derived a closed-form solution for the break-even yield on inflation-indexed bonds for a constant relative-risk averse investor. We now compute the actual value of the break-even yield using our dataset of monthly asset returns and inflation rates from the period January 1985 to October 1999.

In Figure 4 we consider the break-even yield as a function of inflation, given the historically estimated parameters of Section 6. The slope of the function is negative: an increase in inflation reduces the break-even yield. Investors are willing to give up a portion of the interest rate in return for additional protection against inflation. The slope of $r^{*}$ as a function of $\pi$ is equal to -1.00 when the coefficient of relative risk-aversion equals $\alpha=-0.5$. This means that if inflation increases with $1 \%$ the break-even yield decreases with $1 \%$. For other levels of risk aversion the value of this slope hardly changes: the slope is between -1.003 and -0.997 for risk aversion coefficients between $\alpha=-1.5$ and $\alpha=+0.5$.

In Figure 5 we plot the break-even yield itself as a function of the coefficient of relative riskaversion. We observe a strong negative relationship: higher risk aversion reduces the desired return on inflation-indexed bonds. Note that the currently ${ }^{3}$ observed return on inflation-indexed bonds of $3.875 \%$ is consistent with $\alpha=-0.64$. We also studied horizon effects. Fixing the inflation-rate, investors with a longer horizon desire a higher break-even yield, however the differences are negligible. Furthermore, we considered the effect of the correlation between the available assets and the inflation rate on the break-even yield. Higher correlations increase the break-even yield, however, the differences are negligible for reasonable values.

We conclude that the break-even yield is fairly robust to changes in the investor's horizon and the correlation between the asset returns and the inflation rate. The current level of the inflation rate and the risk aversion coefficient of the investor are the main factors driving the demand for inflation-indexed bonds. In order to induce investors to buy inflation-indexed bonds in periods of decreasing inflation, the offered interest rate clearly has to rise. Hence, in this relatively simple model we find motivation for a negative relationship between the real rate of return and the inflation rate, as found in empirical studies (see, e.g. Fama and Gibbons 1982 and Marshall 1992). An interesting topic for further research is to analyse the demand for inflation-linked bonds in an equilibrium framework. We believe that our analysis is an important step in this direction.

\footnotetext{
${ }^{3}$ December 1999 .
} 


\section{Conclusions}

We have formulated the individual portfolio problem in real terms, given uncertainty about future inflation. We believe that this formulation is more adequate and presents the true essence of investment decisions. An investor cares about future purchasing power in real terms rather than in nominal figures. We have solved the optimal portfolio choice problem analytically and shown that optimal portfolios are non-myopic, in contrast to the results of Merton (1969) in a nominal economy.

Only for log-investors or when all assets contain an inflation premium Merton's solution coincides with the optimal portfolio weights derived in this paper. In other cases the investor behaves nonmyopic and additionally invests in an inflation-hedge portfolio. The hedging demand depends on the correlation between asset returns and inflation, the rate of mean-reversion, risk-aversion, and the investor's horizon. In an economy where none of the assets provide inflation-compensation in the drift rate, a change of the inflation rate does not directly influence portfolio choice due to the absence of substitution effects.

When inflation-indexed securities are available or other assets provide partial compensation for inflation, then substitution effects will drive the demand for hedging. We demonstrated that the portfolio weights change continuously, with every small change of the inflation, due to the partial predictability of the mean reverting inflation process. Based on estimated parameters from the recent period 1985-1999, we find that the partial compensation for inflation provided by short-term interest rates has a relatively small impact on portfolio choice. The availability of inflation-indexed securities does have a substantial effect on portfolio choice, especially in periods of high inflation.

The closed-form solutions of our real investment model allow us to investigate the minimal return required by investors on inflation-indexed securities. The inflation rate and the coefficient of risk aversion are the major factors that influence the minimal return. Investors with higher risk aversion accept lower returns on inflation-protected securities, due to their higher hedgingdemand. Regardless of risk aversion, an increase of the inflation rate with $1 \%$ tends to decrease the desired return on inflation-indexed bonds with $1 \%$. Our model indicates that issuers of inflation-indexed bonds are forced to increase real rates of return in times of decreasing inflation, in order to create a market for their product. An interesting topic is to analyse the demand for inflation-linked bonds in an equilibrium framework. We believe that our results are an important step in this direction. A general equilibrium analysis is left for future research. 


\section{A Explicit Solutions for System of Ricatti Equations}

We consider the following stochastic processes in the economy:

$$
\begin{aligned}
& d \pi=\beta(\bar{\pi}-\pi) d t+\sigma_{\pi} d Z_{\pi}, \\
& \frac{d M}{M}=\left(\lambda_{0} \pi+r\right) d t+\sigma_{B} d Z_{B}, \\
& \frac{d P_{i}}{P_{i}}=\left(\lambda_{i} \pi+\mu_{i}\right) d t+\sigma_{i} d Z_{i}, \text { for } \forall i \in\{1 . . I\}, \\
& d Z_{B} d Z_{\pi}=0 .
\end{aligned}
$$

The stochastic process for the price inflation $\pi(t)$ in (47) is an Ornstein-Uhlenbeck process, which is mean reverting to the long run mean $\bar{\pi}$ (if $\beta>0$ ). The instantaneous risk free rate on the money market account in (48) is equal to the sum of the inflation rate $\pi(t) d t$ and the stochastic real rate of return $\left(\lambda_{0}-1\right) \pi(t) d t+r d t+\sigma_{B} d Z_{B}$. The other risky assets follow a geometric Brownian motion with drift $\left(\lambda_{i} \pi+\mu_{i}\right) d t$. The asset returns can be correlated with the inflation rate and the real interest rate.

The real asset value $X(t)$ of the investor evolves according to the following stochastic differential equation:

$$
\begin{aligned}
d X= & \left(\sum_{i=1}^{I} w_{i}\left(\left(\lambda_{i}-\lambda_{0}\right) \pi+\mu_{i}-r\right)\right) X d t+r X d t+\left(\lambda_{0}-1\right) \pi X d t \\
& +\sum_{i=1}^{I} w_{i} X\left(\sigma_{i} d Z_{i}-\sigma_{B} d Z_{B}\right)+\sigma_{B} X d Z_{B}
\end{aligned}
$$

Given the objective (14) in terms of real wealth $X(t)$, the HJB-equation for the value function $J(X(t), \pi(t), t)$ is:

$$
\begin{aligned}
0= & \max _{\{c, w\}} \phi^{J}(c, w ; X, \pi, t) \\
= & J_{t}+\left(w^{\prime}\left(\left(\lambda-\iota \lambda_{0}\right) \pi+\mu-\iota r\right)\right) X J_{X}+r X J_{X}+\left(\lambda_{0}-1\right) \pi X J_{X}+\beta(\bar{\pi}-\pi) J_{\pi} \\
& +\frac{1}{2} w^{\prime} \widehat{\Omega} w X^{2} J_{X X}+\frac{1}{2} \sigma_{B}^{2} X^{2} J_{X X}+\frac{1}{2} \sigma_{\pi}^{2} J_{\pi \pi}+w^{\prime}\left(\sigma_{I B}-\iota \sigma_{B}^{2}\right) X^{2} J_{X X} \\
& +w^{\prime}\left(\sigma_{I \pi}-\iota \sigma_{B \pi}\right) X J_{X \pi}+\sigma_{B \pi} X J_{X \pi}
\end{aligned}
$$

The first order conditions for optimality yield:

$$
w^{*}=-\widehat{\Omega}^{-1}\left(\sigma_{I B}-\iota \sigma_{B}^{2}\right)-\widehat{\Omega}^{-1}\left(\left(\lambda-\iota \lambda_{0}\right) \pi+\mu-\iota r\right) \frac{J_{X}}{X J_{X X}}-\widehat{\Omega}^{-1}\left(\sigma_{I \pi}-\iota \sigma_{B \pi}\right) \frac{J_{X \pi}}{X J_{X X}}
$$


Substituting $w^{*}$ back into the HJB-equation we obtain:

$$
\begin{aligned}
0= & J_{t}+r X J_{X}+\left(\lambda_{0}-1\right) \pi X J_{X}+\beta(\bar{\pi}-\pi) J_{\pi}+\frac{1}{2} \sigma_{\pi}^{2} J_{\pi \pi}+\frac{1}{2} \sigma_{B}^{2} X^{2} J_{X X} \\
& -\frac{1}{2}\left(\left(\lambda-\iota \lambda_{0}\right) \pi+\mu-\iota r\right)^{\prime} \widehat{\Omega}^{-1}\left(\left(\lambda-\iota \lambda_{0}\right) \pi+\mu-\iota r\right) \frac{J_{X}^{2}}{J_{X X}} \\
& -\frac{1}{2}\left(\sigma_{I \pi}-\iota \sigma_{B \pi}\right)^{\prime} \widehat{\Omega}^{-1}\left(\sigma_{I \pi}-\iota \sigma_{B \pi}\right) \frac{J_{X \pi}^{2}}{J_{X X}}-\left(\left(\lambda-\iota \lambda_{0}\right) \pi+\mu-\iota r\right)^{\prime} \widehat{\Omega}^{-1}\left(\sigma_{I \pi}-\iota \sigma_{B \pi}\right) \frac{J_{X} J_{X \pi}}{J_{X X}} \\
& -\frac{1}{2}\left(\sigma_{I B}-\iota \sigma_{B}^{2}\right)^{\prime} \widehat{\Omega}^{-1}\left(\sigma_{I B}-\iota \sigma_{B}^{2}\right) X^{2} J_{X X}+\sigma_{B \pi} X J_{X \pi} \\
& -\left(\sigma_{I B}-\iota \sigma_{B}^{2}\right)^{\prime} \widehat{\Omega}^{-1}\left(\sigma_{I \pi}-\iota \sigma_{B \pi}\right) X J_{X \pi}-\left(\left(\lambda-\iota \lambda_{0}\right) \pi+\mu-\iota r\right)^{\prime} \widehat{\Omega}^{-1}\left(\sigma_{I B}-\iota \sigma_{B}^{2}\right) X J_{X}
\end{aligned}
$$

This HJB-equation is a non-linear second order partial differential equation for the value function $J(X, \pi, t)$, subject to the boundary condition:

$$
J(X(T), \pi(T), T)=\frac{1}{\alpha}(X(T))^{\alpha}
$$

We propose the following functional form for the value function:

$$
\begin{aligned}
& J(X(t), \pi(t), t)=\Phi(\pi(t), t) \frac{1}{\alpha}(X(t))^{\alpha} \\
& \Phi(\pi(t), t)=\exp \left(A(T-t)+B(T-t) \pi(t)+\frac{1}{2} C(T-t) \pi(t)^{2}\right)
\end{aligned}
$$

If we substitute the proposal (55) into the HJB equation, we obtain the following ordinary differential equation:

$$
\begin{aligned}
0= & \frac{1}{\alpha}\left[-A^{\prime}(\tau)-B^{\prime}(\tau) \pi-\frac{1}{2} C^{\prime}(\tau) \pi^{2}\right]+r+\left(\lambda_{0}-1\right) \pi \\
& -\frac{1}{2} \frac{1}{(\alpha-1)} H_{11} \pi^{2}-\frac{1}{(\alpha-1)} H_{12} \pi-\frac{1}{2} \frac{1}{(\alpha-1)} H_{22}-\frac{1}{2}(\alpha-1) H_{33} \\
& -\frac{1}{2} \frac{1}{(\alpha-1)}[B(\tau)+C(\tau) \pi]^{2} H_{44}-\pi H_{13}-H_{23}-[B(\tau)+C(\tau) \pi]\left(H_{34}-\sigma_{B \pi}\right) \\
& -\left[\frac{1}{(\alpha-1)} \pi H_{14}+\frac{1}{(\alpha-1)} H_{24}\right](B(\tau)+C(\tau) \pi)+\frac{1}{2} \sigma_{B}^{2}(\alpha-1) \\
& +\frac{1}{\alpha} \beta(\bar{\pi}-\pi)(B(\tau)+C(\tau) \pi)+\frac{1}{2} \frac{1}{\alpha} \sigma_{\pi}^{2}\left[C(\tau)+B(\tau)^{2}+2 B(\tau) C(\tau) \pi+C(\tau)^{2} \pi^{2}\right]
\end{aligned}
$$


where

$$
\begin{aligned}
\tau & =T-t, \\
H_{11} & =\left(\lambda-\iota \lambda_{0}\right)^{\prime} \widehat{\Omega}^{-1}\left(\lambda-\iota \lambda_{0}\right) \\
H_{12} & =\left(\lambda-\iota \lambda_{0}\right)^{\prime} \widehat{\Omega}^{-1}(\mu-\iota r) \\
H_{13} & =\left(\lambda-\iota \lambda_{0}\right)^{\prime} \widehat{\Omega}^{-1}\left(\sigma_{I B}-\iota \sigma_{B}^{2}\right) \\
H_{14} & =\left(\lambda-\iota \lambda_{0}\right)^{\prime} \widehat{\Omega}^{-1}\left(\sigma_{I \pi}-\iota \sigma_{B \pi}\right) \\
H_{22} & =(\mu-\iota r)^{\prime} \widehat{\Omega}^{-1}(\mu-\iota r) \\
H_{23} & =(\mu-\iota r)^{\prime} \widehat{\Omega}^{-1}\left(\sigma_{I B}-\iota \sigma_{B}^{2}\right) \\
H_{24} & =(\mu-\iota r)^{\prime} \widehat{\Omega}^{-1}\left(\sigma_{I \pi}-\iota \sigma_{B \pi}\right) \\
H_{33} & =\left(\sigma_{I B}-\iota \sigma_{B}^{2}\right)^{\prime} \widehat{\Omega}^{-1}\left(\sigma_{I B}-\iota \sigma_{B}^{2}\right) \\
H_{34} & =\left(\sigma_{I B}-\iota \sigma_{B}^{2}\right)^{\prime} \widehat{\Omega}^{-1}\left(\sigma_{I \pi}-\iota \sigma_{B \pi}\right) \\
H_{44} & =\left(\sigma_{I \pi}-\iota \sigma_{B \pi}\right)^{\prime} \widehat{\Omega}^{-1}\left(\sigma_{I \pi}-\iota \sigma_{B \pi}\right)
\end{aligned}
$$

After some rearranging we are left with the following system of ordinary differential equations:

$$
\begin{aligned}
& C^{\prime}(\tau)=a+b C(\tau)+c C(\tau)^{2}, \\
& B^{\prime}(\tau)=d+\frac{1}{2} b B(\tau)+f C(\tau)+c B(\tau) C(\tau), \\
& A^{\prime}(\tau)=g+f B(\tau)+\frac{1}{2} h C(\tau)+\frac{1}{2} c B(\tau)^{2},
\end{aligned}
$$

where

$$
\begin{aligned}
a & =-\frac{\alpha}{(\alpha-1)} H_{11}, \quad b=-2 \beta-2 \frac{\alpha}{(\alpha-1)} H_{14}, \quad c=\sigma_{\pi}^{2}-\frac{\alpha}{(\alpha-1)} H_{44} \\
d & =-\alpha H_{13}-\frac{\alpha}{(\alpha-1)} H_{12}+\alpha\left(\lambda_{0}-1\right), \quad f=-\alpha\left(H_{34}-\sigma_{B \pi}\right)+\beta \bar{\pi}-\frac{\alpha}{(\alpha-1)} H_{24}, \\
g & =\alpha r-\frac{1}{2} \frac{\alpha}{(\alpha-1)} H_{22}-\frac{1}{2} \alpha(\alpha-1) H_{33}-\alpha H_{23}+\frac{1}{2} \alpha(\alpha-1) \sigma_{B}^{2}, h=\sigma_{\pi}^{2}
\end{aligned}
$$

This system of ordinary non-linear differential equations (59) is the well-known system of Ricatti equations and can be solved recursively.

First we consider the case where none of the available assets provides inflation protection, i.e. $\lambda=0$ and $\lambda_{0}=0$. In this case $H_{11}=H_{12}=H_{13}=H_{14}=0$ and $a=b=0$ holds. Consequently, the solution of the first Ricatti equation is trivial: $C(\tau)=0$. The functions $B(\tau)$ and $A(\tau)$ are given by:

$$
\begin{aligned}
& B(\tau)=\frac{\alpha}{\beta}\left(e^{-\beta \tau}-1\right) \\
& A(\tau)=\left(g-f \frac{\alpha}{\beta}+\frac{1}{2} c\left(\frac{\alpha}{\beta}\right)^{2}\right) \tau+\frac{\alpha}{\beta^{2}}\left(c\left(\frac{\alpha}{\beta}\right)-f\right) e^{-\beta \tau}-\left(\frac{1}{2} \frac{\alpha}{\beta}\right)^{2} \frac{c}{\beta} e^{-2 \beta \tau}+\frac{\alpha}{\beta^{2}}\left(f-\frac{3}{4} \frac{\alpha}{\beta} c\right)
\end{aligned}
$$

Second, we consider the case where all of the assets provides inflation protection, i.e. $\lambda=1$ and $\lambda_{0}=1$. In this case $a=b=0$ again holds and additionally $d=0$. Consequently, the solutions of 
the first and the second Ricatti equations are both zero: $C(\tau)=B(\tau)=0$. The expression for $A(\tau)$ is: $A(\tau)=g \tau$. We conclude that if all assets have an inflation premium, the price inflation $\pi(t)$ has no influence on the adjusted fund value $X(t)$ and hence the value function $J(X, \pi, t)$ is independent of the inflation rate.

Third, we consider the intermediate case where at least one asset provides protection against inflation $\left(\lambda \neq 0\right.$ or $\left.\lambda_{0} \neq 0\right)$, but not all of the assets. The mathematical form of the solution now depends on the discriminant $q$ of the Riccati equation for $C(\tau)$ :

$$
q=b^{2}-4 a c=4 \beta^{2}+4 \frac{\alpha^{2}}{(\alpha-1)^{2}}\left(H_{14}^{2}-H_{11} H_{44}\right)+4 \frac{\alpha}{(\alpha-1)}\left(\sigma_{\pi}^{2} H_{11}-2 \beta H_{14}\right)
$$

The solution for $C(\tau)$ and $B(\tau)$ in the case $q>0$ is:

$$
\begin{aligned}
C(\tau)= & \frac{2 a\left(1-e^{-\eta \tau}\right)}{2 \eta-(b+\eta)\left(1-e^{-\eta \tau}\right)} \\
B(\tau)= & {\left[\left(-2 d(b+\eta)-\frac{f}{c}(b+\eta)^{2}+2 \frac{f}{c} b(b+\eta)\right)\left(e^{-\eta \tau / 2}-1\right)\right.} \\
& \left.-\left(\frac{f}{c}(\eta-b)(\eta+b)+2 d(b-\eta)\right)\left(e^{\eta \tau / 2}-1\right)\right] \frac{e^{-\eta \tau / 2}}{\eta\left[2 \eta-(b+\eta)\left(1-e^{-\eta \tau}\right)\right]}
\end{aligned}
$$

where $\eta=\sqrt{b^{2}-4 a c}$. The function $B(\tau)$ can be reformulated as follows:

$$
B(\tau)=\frac{2(2 a f-b d)}{\eta} \frac{\left(1-e^{-\eta \tau / 2}\right)^{2}}{\left[2 \eta-(b+\eta)\left(1-e^{-\eta \tau}\right)\right]}+\frac{2 d\left(1-e^{-\eta \tau}\right)}{\left[2 \eta-(b+\eta)\left(1-e^{-\eta \tau}\right)\right]},
$$

The solution for an economy with an inflation protected security is a special case of (64) and (65), if we substitute $r=r^{*}$ and $\sigma_{B}^{2}=0$. A remarkable feature of the solution is that $\Phi(\pi(t), t)$, and hence the value function, might reach infinity in finite time. Kim and Omberg (1996) refer to these cases as nirvana solutions. The conditions in (67) should be satisfied for the occurrence of nirvana solution in the case $q>0$ (i.e. these conditions are necessary, but not sufficient for nirvana solutions).

$$
a c>0, b>0 \text { and } T>\frac{1}{\eta} \ln \left(\frac{b+\eta}{b-\eta}\right)
$$

In the special case $q=0$ and $b \neq 0$ the solution for $C(\tau)$ and $B(\tau)$ is (see Abramowitz and Stegun 1964):

$$
\begin{aligned}
& C(\tau)=-\frac{b}{2 c}-\frac{1}{c\left(\tau-\frac{2}{b}\right)} \\
& B(\tau)=-\frac{1}{2} \frac{(2 a f-b d) \tau^{2}}{b\left(\tau-\frac{2}{b}\right)}-\frac{2 d \tau}{b\left(\tau-\frac{2}{b}\right)}
\end{aligned}
$$

The conditions in (70) should be satisfied for the occurrence of nirvana solutions in this case:

$$
b>0 \text { and } T>2 / b
$$


When $q=0$ and additionally $b=0$ we have $c=0$ and the solution for $C(\tau)$ and $B(\tau)$ in this case is given by:

$$
\begin{aligned}
& C(\tau)=a \tau \\
& B(\tau)=d \tau+\frac{1}{2} a f \tau^{2}
\end{aligned}
$$

and nirvana solutions do not occur.

Finally, if $q<0$ then nirvana always occurs, and the solution for $C(\tau)$ and $B(\tau)$ is:

$$
\begin{aligned}
C(\tau) & =\frac{\eta}{2 c} \tan \left[\frac{\eta}{2} \tau+k\right]-\frac{b}{2 c} \\
B(\tau) & =\left(\frac{2 d c-f b}{\eta c}\right) \frac{\sin \left[\frac{\eta}{2} \tau+k\right]-\sin [k]}{\cos \left[\frac{\eta}{2} \tau+k\right]}-\frac{f}{c} \frac{\cos \left[\frac{\eta}{2} \tau+k\right]-\cos [k]}{\cos \left[\frac{\eta}{2} \tau+k\right]}
\end{aligned}
$$

where $\eta=\sqrt{4 a c-b^{2}}, k=\arctan \left[\frac{b}{\eta}\right]$.

\section{B Nirvana Solutions in a Two-Asset Economy}

In this appendix, we consider the occurrence of nirvana-type solutions. In Appendix A we discussed that for $q>0$ nirvana solutions occur if

$$
a c>0, b>0 \text { and } T>\frac{1}{\eta} \ln \left(\frac{b+\eta}{b-\eta}\right) .
$$

Furthermore, if $q<0$ then every solution is a nirvana solution. For the solutions in the special case $q=0$ we refer to Appendix A.

We show that in the case of one risky asset (e.g. stocks) and an inflation-indexed bond nirvana solutions cannot occur.

$$
\begin{aligned}
a & =\frac{\alpha}{1-\alpha} \iota^{\prime} \Omega^{-1} \iota=\frac{\alpha}{1-\alpha} \frac{1}{\sigma_{S}^{2}}<0 \text { if } \alpha<0 \\
b & =-2\left(\beta+\frac{\alpha}{1-\alpha} \rho_{S \pi} \frac{\sigma_{\pi}}{\sigma_{S}}\right) \\
c & =\sigma_{\pi}^{2}+\frac{\alpha}{1-\alpha} \rho_{S \pi}^{2} \sigma_{\pi}^{2}
\end{aligned}
$$

where $\sigma_{S}^{2}$ denote the instantaneous variance of the risky asset, $\rho_{S \pi}$ denotes the correlation between returns on the risky asset and inflation, and $\sigma_{\pi}^{2}$ denotes the instantaneous variance of inflation. Assume first that $q>0$, then nirvana solutions are only possible if $c<0$ (since $a<0$ ). Hence

$$
\sigma_{\pi}^{2}+\frac{\alpha}{1-\alpha} \rho_{S \pi}^{2} \sigma_{\pi}^{2}<0 \Longleftrightarrow \rho_{S \pi}^{2}>1-\frac{1}{\alpha}
$$


However, since $\alpha<0$ this can never occur, all normal solutions are well-behaved in this case. Of course, nirvana solutions may occur when $q<0$. However, we will show that this cannot happen for the two assets case studied here. We have

$$
q=b^{2}-4 a c=4 \beta^{2}-4 \frac{\alpha}{1-\alpha}\left(\frac{\sigma_{\pi}^{2}}{\sigma_{S}^{2}}-2 \beta \frac{\rho_{S \pi} \sigma_{\pi}}{\sigma_{S}}\right)
$$

First, consider $q$ as a parabola in $\beta$. We may write $q(\beta)=c_{0}+c_{1} \beta+c_{2} \beta^{2}$. Then, since $c_{2}=4>0$, we conclude that $q(\beta)$ is convex. Furthermore, the discriminant is given by:

$$
D=c_{1}^{2}-4 c_{0} c_{2}=64\left(\frac{\sigma_{\pi}^{2}}{\sigma_{S}^{2}}\right)\left(\left(\frac{\alpha}{1-\alpha}\right)^{2} \rho_{S \pi}^{2}+\frac{\alpha}{1-\alpha}\right)
$$

Hence $D>0$ only if

$$
\rho_{S \pi}^{2}>1-\frac{1}{\alpha}
$$

This can never occur, since $\alpha<0$, and hence $q(\beta)>0$, for all $\beta$.

Similarly for $q(s)=c_{0}+c_{1} s+c_{2} s^{2}$, with $s=\frac{\sigma_{\pi}}{\sigma_{S}}$ we have a convex parabola, since $c_{2}=-4 \frac{\alpha}{1-\alpha}>$ 0 (if $\alpha<0$ ) furthermore

$$
D=c_{1}^{2}-4 c_{0} c_{2}=64 \beta^{2}\left(\left(\frac{\alpha}{1-\alpha}\right)^{2} \rho_{S \pi}^{2}+\frac{\alpha}{1-\alpha}\right)
$$

Hence $D>0$ only if

$$
\rho_{S \pi}^{2}>1-\frac{1}{\alpha}
$$

This can never occur, since $\alpha<0$, and hence $q(s)>0$, for all $s$.

Finally we consider $q\left(\rho_{S \pi}\right)$ as a function of the correlation between the return on the risky asset and inflation: $q(\rho)=4 \beta^{2}-4 \frac{\alpha}{1-\alpha} s^{2}+8 \beta \frac{\alpha}{1-\alpha} s \rho$. Hence, $q(\rho)<0$ if and only if

$$
\rho>\frac{1}{2} \frac{s}{\beta}+\frac{1}{2} \frac{\beta}{s}\left(1-\frac{1}{\alpha}\right)>1, \text { if } \alpha<0
$$

We may conclude that in the two-asset case nirvana solutions never occur. 


\section{References}

Abramowitz and Stegun, 1964, Handbook of Mathematical Functions with Formulas, Graphs, and Mathematical Tables, Wiley, New York.

Barnes, M., J.H. Boyd, and B.D. Smith, 1999, "Inflation and Asset Returns", European Economic Review, 43, 737-754.

Bodie, Z., 1976, "Common Stocks as a Hedge Against Inflation", Journal of Finance, 31, 459470.

Bodie, Z., 1990, "Inflation insurance", Journal of Risk \& Insurance, 57, 634-645.

Bos, C.S., P.H. Franses and M. Ooms, 1999, "Long Memory and Level Shifts: Re-Analyzing Inflation Rates", Empirical Economics, 24, 427-449.

Brennan, M.J., E.S. Schwartz, and R. Lagnado, 1997, "Strategic Asset Allocation", Journal of Economic Dynamics and Control, 21, 1377-1403.

Fama, E.F., 1981, "Stock Returns, Real Activity, Inflation, and Money", American Economic Review, 77, 545-565.

Fama, E.F. and M. Gibbons, 1982, "Inflation, Real Returns and Capital Investment", Journal of Monetary Economics, 9, 297-323.

Fama, E.F. and G.W. Schwert, 1977, "Asset Returns and Inflation”, Journal of Financial Economics, 5, 115-146.

Fisher, I., 1930, The Theory of Interest, MacMillan: New York.

Geltner, D.M., 1991, "Smoothing in Appraisal-Based Returns", Journal of Real Estate Finance and Economics, 4, 327-345.

Geske, R. and R. Roll, 1983, "The Monetary and Fiscal Linkages between Stock Returns and Inflation", Journal of Finance, 38, 1-33.

Goetzmann, W. and R. Ibbotson, 1990, "The Performance of Real Estate as an Asset Class", Journal of Applied Corporate Finance, 3, 65-76.

Gultekin, N.B., 1983, "Stock Market Returns and Inflation: Evidence from Other Countries", Journal of Finance, 38, 49-65.

Hassler, U. and J. Wolters, 1995, "Long Memory in Inflation Rates: International Evidence", Journal of Business \& Economics Statistics, 13, 37-46.

Ibbotson, R. and L. Siegel, 1984, "Real Estate Returns: A Comparison with Other Investments", The Journal of the American Real Estate and Urban Economics Association, 12, 219-242.

Kim, T.S. and E. Omberg, 1996, "Dynamic Nonmyopic Portfolio Behavior", Review of Financial Studies, 9, 141-161.

Marshall, D., 1992, "Inflation and Asset Returns in a Monetary Economy", Journal of Finance, 47, 1315-1342.

McFall Lamm Jr, R., 1998, "Asset Allocation Implications of Inflation Protection Securities", Journal of Portfolio Management, 24, 93-100.

Merton R.C., 1969, "Lifetime Portfolio Selection under Uncertainty: The Continuous-Time Case", Review of Economics and Statistics, 51, 247-257. 
Merton R.C., 1971, "Optimum Consumption and Portfolio Rules in a Continuous-Time Model”, Journal of Economic Theory, 3, 373-413.

Øxendal B., 1998, Stochastic Differential Equations (5th ed.), Springer Verlag, Berlin.

Samuelson P.A., 1969, "Lifetime Portfolio Selection by Dynamic Stochastic Programming", Review of Economics and Statistics, 51, 239-246.

Stulz, R., 1986, "Asset Pricing and Expected Inflation", Journal of Finance, 41, 209-224. 
Table 1: Mean-Reversion of Inflation-Rates, 1985-1999

\begin{tabular}{cccc}
\hline$\Delta \pi_{t}=$ & 0.018 & $-0.575 \pi_{t-1}+e_{t}$, \\
Adj. $R^{2}=0.28$, & $\sigma_{e}=0.030$ & \\
\hline
\end{tabular}

Estimated coefficients of the regression $\Delta \pi_{t}=\alpha_{0}+\alpha_{1} \pi_{t-1}+\epsilon_{t}$, where $\pi_{t}$ denotes the monthly US inflation-rate. The t-statistics are denoted between brackets.

Table 2: Summary Statistics for Inflation and Asset Returns, 1985-1999

\begin{tabular}{lrrrr}
\hline \hline & Mean & Std. Dev. & Skewness & Excess Kurtosis \\
\hline CPI inflation rate & 3.14 & 2.16 & 0.003 & 3.66 \\
CP rate & 5.80 & 1.58 & 0.07 & -0.68 \\
1-Yr T-Bills & 5.58 & 1.38 & 0.09 & -0.72 \\
E-REITs & 9.33 & 41.68 & -0.52 & 3.36 \\
Bonds & 10.58 & 32.55 & 0.23 & 0.59 \\
S\&P 500 & 13.94 & 38.92 & -0.85 & 3.77 \\
\hline
\end{tabular}

Statistics are based on continuously compounded returns. The asset classes considered are: financial commercial paper rate, 1-year Treasury bills, Equity Real Estate Investment Trusts, Salomon Brothers bond index with bonds with maturity of 10 years and longer, and total returns on the S\&P 500 index. Inflation rates are determined from the Consumer Price Index.

Table 3: Correlations between Asset Returns and Inflation, 1985-1999

\begin{tabular}{lrrrrr}
\hline \hline & CP rate & $1-Y r$ T-Bills & E-REITs & Bonds & S\&̈P 500 \\
\hline CPI inflation rate & 0.31 & 0.31 & -0.04 & 0.03 & -0.13 \\
CP rate & 1.0 & 0.97 & -0.03 & 0.16 & 0.04 \\
1-Yr T-Bills & & 1.0 & -0.03 & 0.18 & 0.01 \\
E-REITs & & & 1.0 & 0.24 & 0.42 \\
Bonds & & & 1.0 & 0.15 \\
Stocks & & & & 1.0 \\
\hline
\end{tabular}

Correlations are based on continuously compounded returns. The asset classes considered are: financial commercial paper rate, 1-year Treasury bills, Equity Real Estate Investment Trusts, Salomon Brothers bond index with bonds with maturity of 10 years and longer, and total returns on the S\&P 500 index. Inflation rates are determined from the Consumer Price Index. 
Table 4: Regression Results of Asset Returns on Inflation, 1985-1999

\begin{tabular}{|c|c|c|c|c|c|c|c|c|c|c|}
\hline & CP rate & & $1-\mathrm{Yr} \mathrm{T-1}$ & & E-REIT & & Bonds & & S\&P 500 & \\
\hline$\gamma_{0}$ & $0.051^{* *}$ & $(14.02)$ & $0.050^{* *}$ & $(16.58)$ & $0.115^{*}$ & $(2.14)$ & $0.089^{*}$ & $(2.39)$ & $0.212^{* *}$ & $(4.82)$ \\
\hline & $0.223^{*}$ & $(2.59)$ & $0.195^{* *}$ & $(2.82)$ & -0.702 & $(-0.46)$ & 0.522 & $(0.51)$ & -2.30 & $(-1.64)$ \\
\hline Adj. $R^{2}$ & 0.09 & & 0.09 & & 0.00 & & 0.00 & & 0.01 & \\
\hline
\end{tabular}

The results are from the regression $R_{t}=\alpha_{0}+\alpha_{1} \pi_{t}+\epsilon_{t}$, where $R_{t}$ denotes the continuously compounded return in month $t$ and $\pi_{t}$ denotes the inflation-rate based on the Consumer Price Index. The t-statistics are denoted between brackets; we use adjusted t-statistics employing the Newey-West variance estimator, to correct for autocorrelated error terms. One asterix $\left(^{*}\right)$ denotes significance at the 0.05 level, (**) denotes significance at the 0.01 level.

Table 5: Occurrence of Nirvana Solutions

\begin{tabular}{llll}
\hline \hline inflation-indexed bond & three assets & four assets & five assets \\
\hline$q$ & 1.34 & 3.66 & 3.67 \\
$a c$ & -0.0018 & -0.68 & -0.69 \\
$b$ & -1.15 & -0.97 & -0.96 \\
\hline inflation-premia & three assets & four assets & five assets \\
\hline$q$ & 1.32 & 1.34 & 1.34 \\
$a c$ & $-9.0 \times 10^{-5}$ & -0.0055 & -0.0055 \\
$b$ & -1.15 & -1.15 & -1.15 \\
\hline
\end{tabular}

In the case of an inflation-indexed bond, none of the assets are allowed to possess an inflation premium. Financial commercial paper rate serves as the money market account. In the three-asset case the investor can additionally invest in a Salomon Brothers bond index with bonds with maturity of 10 years and longer and the S\&P 500 index. The four-asset case contains 1-year Treasury bills as an additional asset class. In the five-asset case the investor may invest in Equity Real Estate Investment Trusts. The inflation premium for 1 -year T-bills equals $\gamma_{1}=0.19$ and for commercial paper $\gamma_{1}=0.22$. 
Table 6: Demand for Inflation-Indexed Bonds

\begin{tabular}{llrrrrr}
\hline \hline & inflation & $0 \%$ & $2.5 \%$ & $5.0 \%$ & $7.5 \%$ & $10.0 \%$ \\
\hline$r^{*}=3.3 \%$ & iib & $-5.1 \%$ & $12.8 \%$ & $30.6 \%$ & $48.4 \%$ & $66.3 \%$ \\
& bonds & $51.1 \%$ & $40.3 \%$ & $29.5 \%$ & $18.7 \%$ & $7.8 \%$ \\
& stocks & $54.0 \%$ & $47.0 \%$ & $39.9 \%$ & $32.9 \%$ & $25.9 \%$ \\
$r^{*}=3.5 \%$ & iib* & $-3.7 \%$ & $14.2 \%$ & $32.0 \%$ & $49.9 \%$ & $67.7 \%$ \\
& bonds & $50.3 \%$ & $39.4 \%$ & $28.6 \%$ & $17.8 \%$ & $7.0 \%$ \\
& stocks & $53.4 \%$ & $46.4 \%$ & $39.4 \%$ & $32.4 \%$ & $25.4 \%$ \\
$r^{*}=3.7 \%$ & iib* & $-2.3 \%$ & $15.6 \%$ & $33.4 \%$ & $51.3 \%$ & $69.1 \%$ \\
& bonds & $49.4 \%$ & $38.6 \%$ & $27.7 \%$ & $16.9 \%$ & $6.1 \%$ \\
& stocks & $52.9 \%$ & $45.6 \%$ & $38.8 \%$ & $31.8 \%$ & $24.8 \%$ \\
\hline
\end{tabular}

The investor has a horizon of 20 years and relative risk-aversion coefficient equal to $\alpha=-1.0$. The asset class bonds is the Salomon Brothers Bond Index and the asset class stocks denotes the S\&P500.

* Inflation-indexed bond.

Figure 1: US Inflation rates

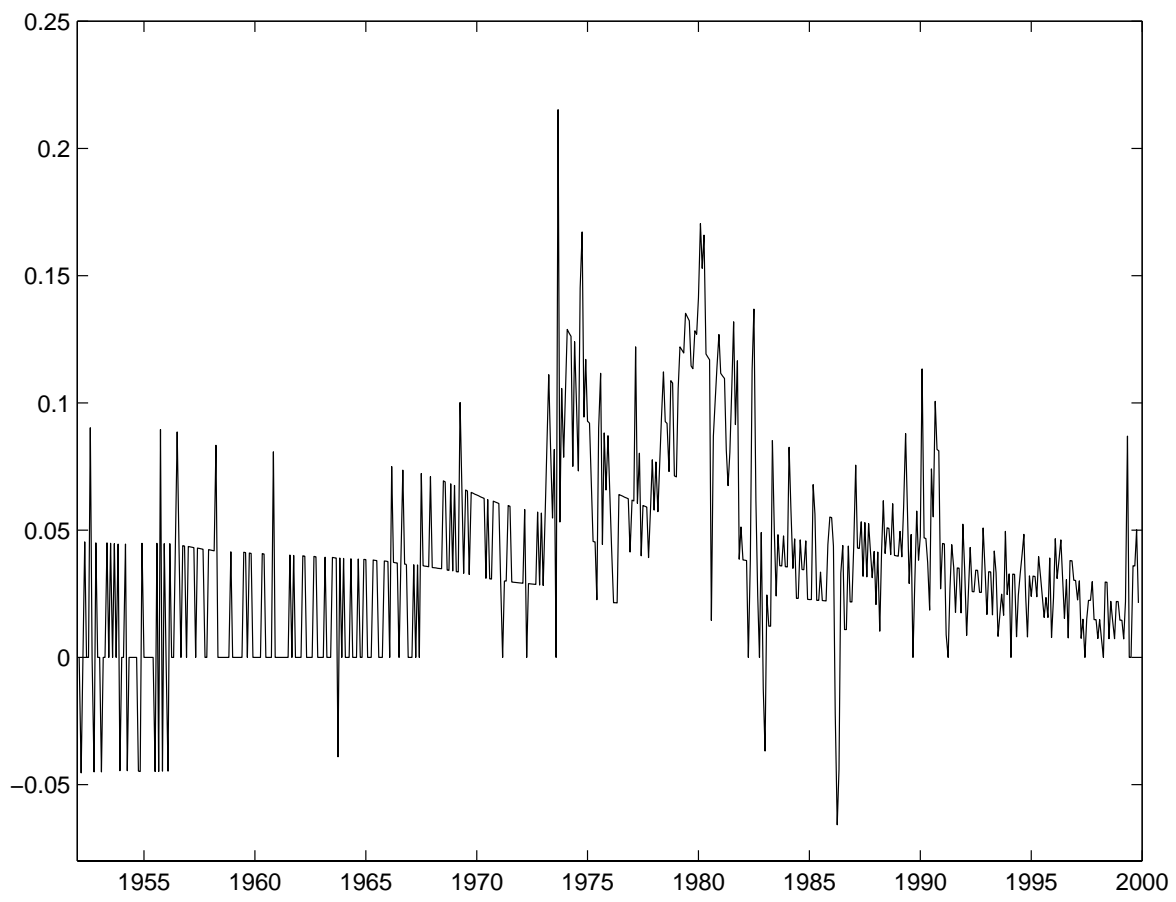

This figure shows the monthly US inflation rates for the period January 1952 through October 1999. The rates are continuously compounded and annualized. 
Figure 2: Fraction Invested in Stocks

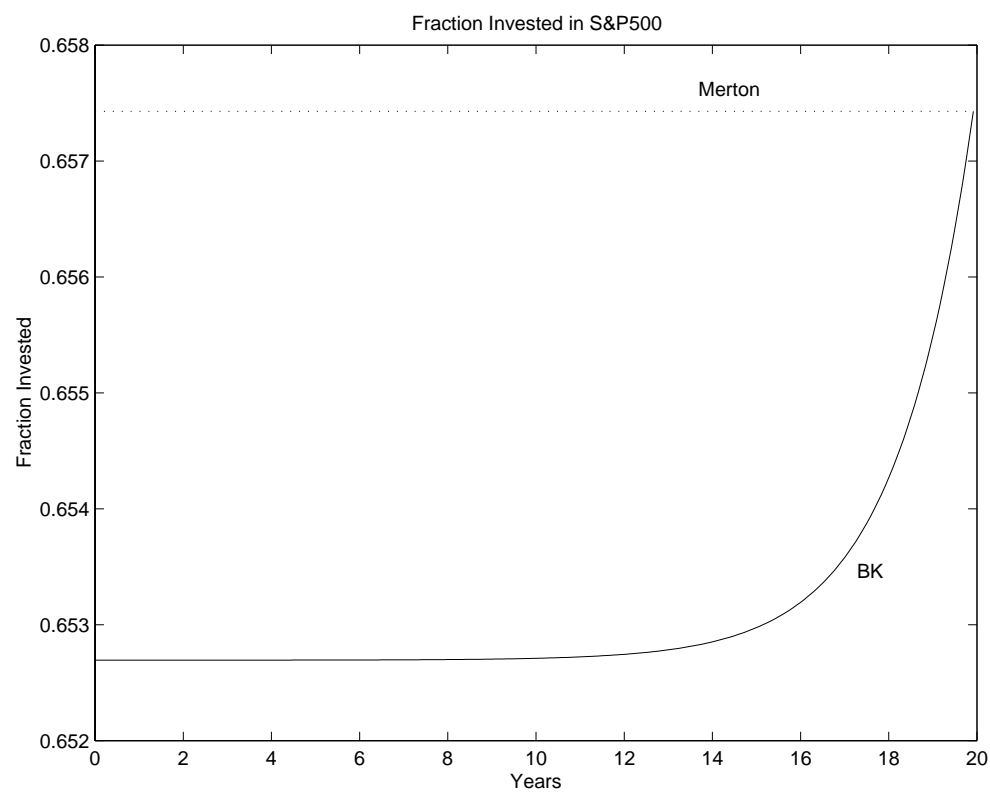

This figure shows the fraction invested in the S\&P500 over the next 20 years for a myopic investor and an investor taking the correlation between asset returns and inflation into account. The coefficient of relative risk-aversion equals $\alpha=-0.5$.

Figure 3: Fraction Invested in Stocks

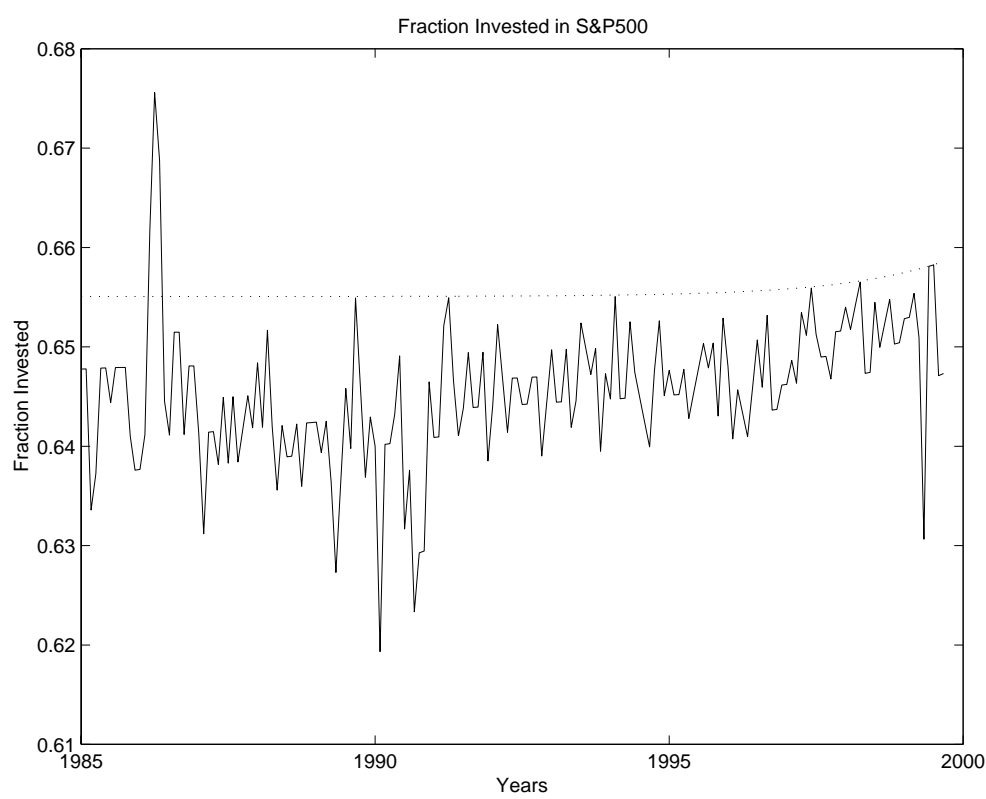

This figure shows the fraction invested in the S\&P500 over the sampling period January 1985-October 1999 for a constant relative risk-averse investor in an economy with and without substitution effects. The coefficient of relative risk-aversion equals $\alpha=-0.5$. 
Figure 4: Break-even Yield on Inflation-Indexed Bond

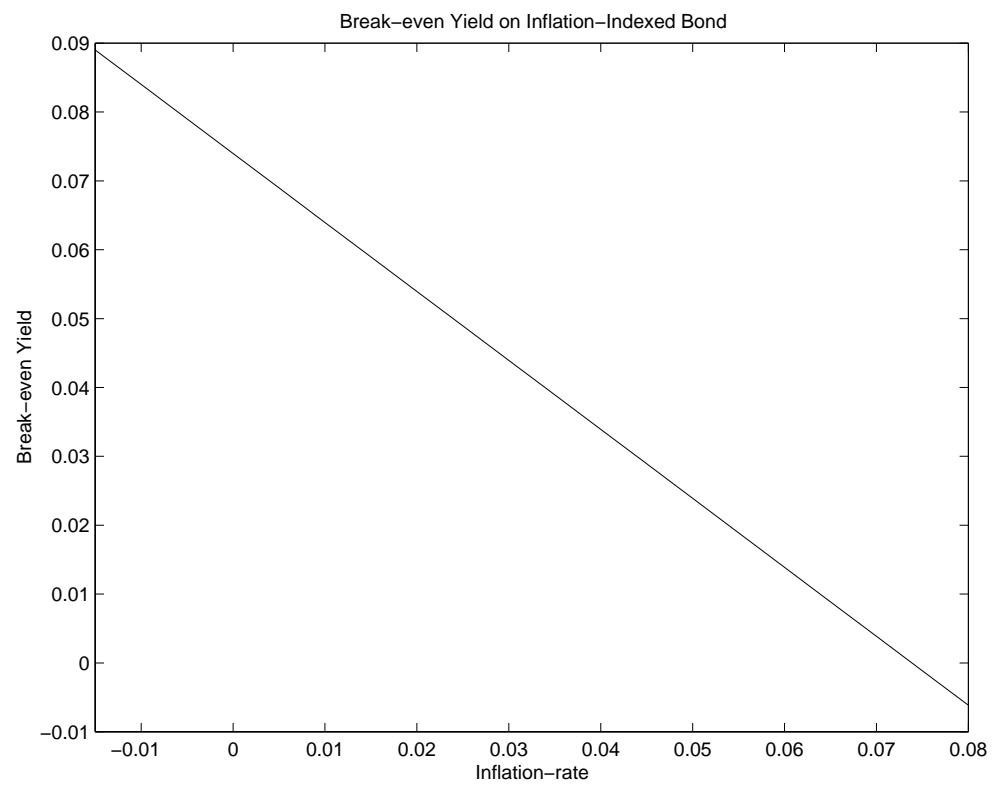

This figure shows the break-even yield as a function of inflation, when the coefficient of relative riskaversion equals $\alpha=-0.5$.

Figure 5: Break-even Yield on Inflation-Indexed Bond

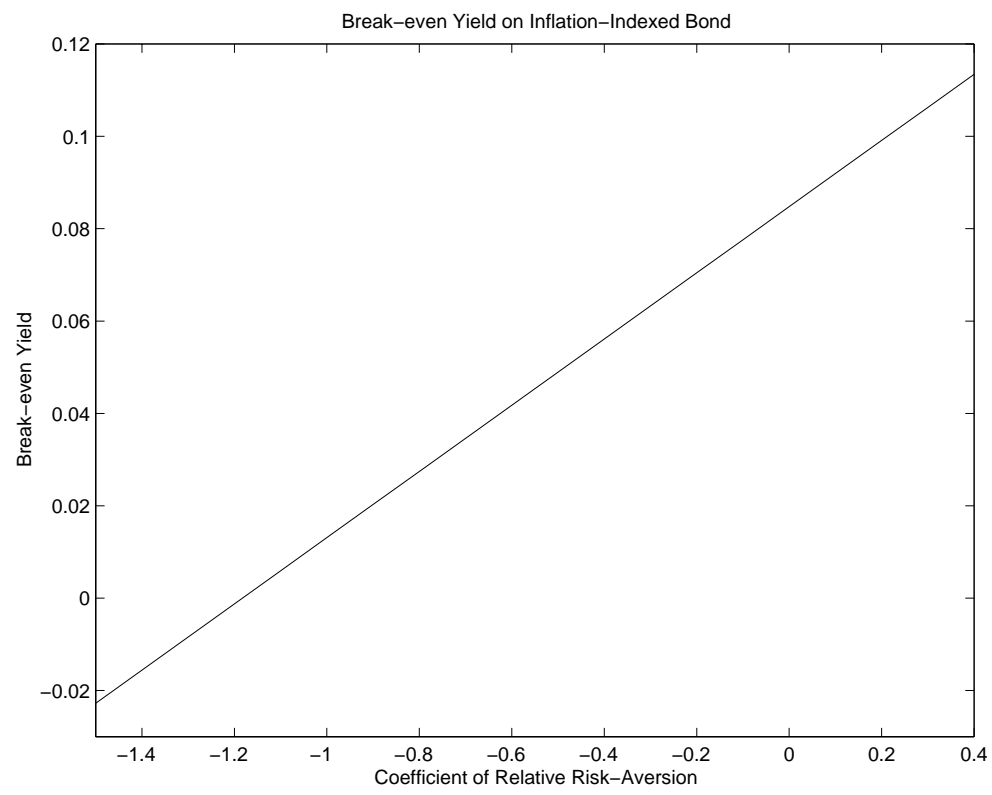

This figure shows the break-even yield as a function of the coefficient of relative risk-aversion, assuming a constant inflation-rate equal to $3 \%$. 